\title{
Two-step values for games with two-level communication structure
}

\author{
Sylvain Béal ${ }^{1} \cdot$ Anna Khmelnitskaya $^{2}$ (1) \\ Philippe Solal ${ }^{3}$
}

(C) The Author(s) 2017. This article is an open access publication

\begin{abstract}
TU games with two-level communication structure, in which a two-level communication structure relates fundamentally to the given coalition structure and consists of a communication graph on the collection of the a priori unions in the coalition structure, as well as a collection of communication graphs within each union, are considered. For such games we introduce two families of two-step values inspired by the two-step procedures staying behind the Owen value (Owen, in: Henn, Moeschlin (eds) Essays in mathematical economics and game theory, Springer, Berlin, pp 76-88, 1977) and the two-step Shapley value (Kamijo in Int Game Theory Rev 11:207-214, 2009) for games with coalition structure. Our approach is based on the unified treatment of several component efficient values for games with communication structure and it generates two-stage solution concepts that apply component efficient values for games
\end{abstract}

The authors are grateful to two anonymous reviewers for valuable comments. The financial support of the first and third authors from research program "DynaMITE: Dynamic Matching and Interactions: Theory and Experiments", Contract ANR-13-BSHS1-0010, and of the second author from RFBR (Russian Foundation for Basic Research) Grant \#16-01-00713 is gratefully acknowledged. The research of the second author was done partially during her stay at the University of Twente whose hospitality is also highly appreciated.

$凶 \quad$ Anna Khmelnitskaya

a.b.khmelnitskaya@utwente.nl

Sylvain Béal

sylvain.beal@univ-fcomte.fr

Philippe Solal

philippe.solal@univ-st-etienne.fr

1 CRESE EA3190, Univ. Bourgogne Franche-Comté, 25000 Besançon, France

2 Saint-Petersburg State University, Faculty of Applied Mathematics, Saint Petersburg, Russia

3 Université Jean Monnet, Saint-Étienne, France 
with communication structure on both distribution levels. Comparable axiomatic characterizations are provided.

Keywords TU game with two-level communication structure - Owen value . Two-step Shapley value · Component efficiency $\cdot$ Deletion link property

Mathematics Subject Classification 91A12 - 91A43

\section{JEL Classification C71}

\section{Introduction}

In classical cooperative game theory it is assumed that any coalition of players may form, and a fair distribution of total rewards amongst the players takes into account capacities of all coalitions. For example, the most prominent solution of cooperative games, the Shapley value (Shapley 1953), assigns to each player as a payoff the average of the player's marginal contributions to his predecessors with respect to all possible linear orderings of the players. However, in many practical situations the collection of feasible coalitions is restricted by some social, economical, hierarchical, communicational, or technical structure. The study of transferable utility (TU) games with limited cooperation introduced by means of coalition structures, or in other terms a priori unions, was initiated in the 1970's first by Aumann and Drèze (1974) and then by Owen (1977). In these papers a coalition structure is given by a partition of the set of players, and both papers introduce as solutions some adaptations of the classical Shapley value to this situation with restricted cooperation. While Aumann and Drèze assume that cooperation is possible only within a priori unions and as a solution they propose the combination of Shapley values in subgames on a priori unions, Owen admits some cooperation between players of different unions. Similar to the Shapley value, the Owen value assigns to each player as a payoff the average of the player's marginal contributions to his predecessors with respect to linear orderings of the players. But in case of Owen not all possible linear orderings are taken into account, but only those in which the players of the same a priori union appear successively. Another model of a game with limited cooperation presented by means of undirected communication graphs was introduced in Myerson (1977). The main assumption of Myerson is that only connected players are able to cooperate, and the Myerson value is given by the Shapley value in the Myerson restricted game, in which the worth of each disconnected coalition is replaced by the sum of the worths of its connected components. Various studies in both directions were done during the last four decades, but mostly either within one model or another. Vázquez-Brage et al. (1996) is the first study that combines both models by considering a TU game endowed with, independent of each other, both a coalition structure and a communication graph on the set of players. For this class of games they propose a solution by applying the Owen value for games with coalition structure to the Myerson restricted game of the game with communication graph.

Another model of a TU game endowed with both a coalition structure and a communication graph, the so-called game with two-level communication structure, is 
considered in Khmelnitskaya (2014). In contrast to Vázquez-Brage et al. (1996), in this model a two-level communication structure relates fundamentally to the given coalition structure and consists of a communication graph on the collection of the a priori unions in the coalition structure, as well as a collection of communication graphs within each union. It is assumed that communication is only possible either among the entire a priori unions or among single players within any a priori union. No communication and therefore no cooperation is allowed between proper subcoalitions, in particular single players, of distinct elements of the coalition structure. ${ }^{1}$ This approach allows to model different network situations, in particular, telecommunication problems, distribution of goods among different cities (countries) along highway networks connecting the cities and local road networks within the cities, or sharing an international river with multiple users but without international firms, i.e., when no cooperation is possible among single users located at different levels along the river, and so on. Communication structures under scrutiny are given by combinations of graphs of different types both undirected-arbitrary graphs and cycle-free graphs, and directed-line-graphs with linearly ordered players, rooted forests and sink forests. The proposed solution concepts reflect a two-stage distribution procedure when, first, a priori unions collect their shares through the upper level bargaining based only on the cumulative interests of all members of every involved entire a priori union, and second, the players collect their individual payoffs through the lower level bargaining over the distribution of the unions' shares within the unions. Following Myerson (1977) it is assumed that cooperation is possible only between connected participants and different combinations of known component efficient values, such as the Myerson value, the position value, the average tree solution, etc., are applied on both communication levels. However, as discussed in Khmelnitskaya (2014), the two-stage distribution procedure based on the application of component efficient values on both levels suffers from severe restrictions in cases when some a priori unions are internally not connected, because each union always has to distribute its total share among the members. Another solution concept for TU games with two-level communication structure introduced by means of undirected graphs, the so-called Owen-type value for games with two-level communication structure, is considered in van den Brink et al. (2016) under a weaker assumption concerning the communication on the level of a priori unions, when on the upper level bargaining between a priori unions, similar as for the Owen value introduced in Owen (1977), one of the a priori unions can be presented by any of its proper subcoalitions. This solution can be seen as an adaptation of the two-step procedure determining the Owen value for games with coalition structure which takes into account the limited cooperation represented by two-level communication structure replacing twice the Shapley value by the Myerson value. ${ }^{2}$

\footnotetext{
1 A similar model, but with other and quite special assumptions concerning the ability of players to cooperate under given communication constraints is also studied in Kongo (2011).

2 An extension of the Owen-type value introduced in van den Brink et al. (2016), when the underlying two-step procedure determining the Owen value for games with coalition structure is replaced by a similar two-step procedure, in which on both steps the application of the Shapley value is replaced by the $\tau$-value, is studied recently in Zhang et al. (2017).
} 
In this paper we assume that a two-level communication structure is given by combinations of graphs of different types, both undirected and directed, and we introduce two families of two-step values for games with two-level communication structure adapting the two-step procedures staying behind two values for games with coalition structure, the Owen value and the two-step Shapley value introduced in Kamijo (2009). Our approach is based on the unified treatment of several component efficient values for games with communication structure and it generates two-stage solution concepts that apply component efficient values for games with communication structure on both distribution levels. In fact the newly introduced family of the Owen-type values is the generalization of the Owen-type value for games with two-level communication structures of van den Brink et al. (2016), when on both communication levels not only the Myerson value, but different component efficient values for games with communication structure can be applied. The incorporation of different solutions for games with communication structure aims not only to enrich the solution concepts for games with two-level communication structure, but it also opens a broad diversity of applications impossible otherwise, because there exists no universal solution concept for games with communication structure that is applicable to the full variety of possible undirected and directed graph structures. Moreover, it allows to choose, depending on types of graph structures under scrutiny, the most preferable, in particular, the most computationally efficient combination of values among others suitable. We provide axiomatic characterizations of the introduced two-step values. These axiomatizations have several common axioms for the both families which allows to compare the twostep values from different families.

The introduced two families of two-step solution concepts may find application in different resource allocation problems with two hierarchical distribution levels. For instance, they may be used for budget allocation within a university, when the budget has to be distributed first among its departments and then among the individuals within each department. An example showing advantages of using the Owen-type value for games with two-level communication structures, in case when on both communication levels only the Myerson value is applied, is discussed in van den Brink et al. (2016).

The rest of the paper is organized as follows. Basic definitions and notation are introduced in Sect. 2. Section 3 provides the uniform approach to several known component efficient values for games with communication structure, which allows also to consider within a unified framework different deletion link properties with respect to the values for games with two-level communication structure. In Sects. 4 and 5 we introduce correspondingly the families of the Kamijo-type and Owen-type values axiomatically and present their explicit formula representations. Section 6 concludes.

\section{Preliminaries}

\subsection{TU games and values}

A cooperative game with transferable utility, or $T U$ game, is a pair $\langle N, v\rangle$, where $N \subset \mathbb{N}$ is a finite set of $n$ players and $v: 2^{N} \rightarrow \mathbb{R}$ is a characteristic function with $v(\emptyset)=0$, assigning to every coalition $S \subseteq N$ of $s$ players its worth $v(S)$. The set of 
TU games with fixed player set $N$ is denoted by $\mathcal{G}_{N}$. For simplicity of notation and if no ambiguity appears we write $v$ when we refer to a TU game $\langle N, v\rangle$. The subgame of a TU game $v \in \mathcal{G}_{N}$ with respect to a player set $T \subseteq N$ is the TU game $\left.v\right|_{T} \in \mathcal{G}_{T}$ defined by $\left.v\right|_{T}(S)=v(S)$ for every $S \subseteq T$. A payoff vector is a vector $x \in \mathbb{R}^{N}$ with $x_{i}$ the payoff to player $i \in N$. A single-valued solution, called a value, is a mapping $\xi: \mathcal{G}_{N} \rightarrow \mathbb{R}^{N}$ that assigns to every finite set $N \subset \mathbb{N}$ and every TU game $v \in \mathcal{G}_{N}$ a payoff vector $\xi(v) \in \mathbb{R}^{N}$. A value $\xi$ is efficient if $\sum_{i \in N} \xi_{i}(v)=v(N)$ for every $v \in \mathcal{G}_{N}$ and $N \subset \mathbb{N}$. The best-known efficient value is the Shapley value (Shapley 1953) given by

$$
S h_{i}(v)=\sum_{S \subseteq N \backslash\{i\}} \frac{s !(n-s-1) !}{n !}(v(S \cup\{i\})-v(S)), \quad \text { for all } i \in N
$$

In the sequel we denote the cardinality of a given set $A$ by $|A|$, along with lower case letters like $n=|N|, m=|M|, n_{k}=\left|N_{k}\right|, s=|S|, c=|C|, c^{\prime}=\left|C^{\prime}\right|$, and so on, and we use the standard notation $x(S)=\sum_{i \in S} x_{i}$ for any $x \in \mathbb{R}^{N}$ and $S \subseteq N$.

\subsection{Games with coalition structure}

A coalition structure, or in other terms a system of a priori unions, on $N \subset \mathbb{N}$ is given by a partition $\mathcal{P}=\left\{N_{1}, \ldots, N_{m}\right\}$ of $N$, i.e., $N_{1} \cup \ldots \cup N_{m}=N$ and $N_{k} \cap N_{l}=\varnothing$ for $k \neq l$. Let $\mathfrak{P}_{N}$ denote the set of all coalition structures on $N$, and let $\mathcal{G}_{N}^{P}=\mathcal{G}_{N} \times \mathfrak{P}_{N}$. A pair $\langle v, \mathcal{P}\rangle \in \mathcal{G}_{N}^{P}$ constitutes a game with coalition structure, or simply $P$-game, on $N$. Remark that $\langle v,\{N\}\rangle$ represents the same situation as $v$ itself. A $P$-value is a mapping $\xi: \mathcal{G}_{N}^{P} \rightarrow \mathbb{R}^{N}$ that assigns to every $N \subset \mathbb{N}$ and every $P$-game $\langle v, \mathcal{P}\rangle \in \mathcal{G}_{N}^{P}$ a payoff vector $\xi(v, \mathcal{P}) \in \mathbb{R}^{N}$. A $P$-value $\xi$ is efficient if $\sum_{i \in N} \xi_{i}(v, \mathcal{P})=v(N)$ for every $v \in \mathcal{G}_{N}^{P}$ and $N \subset \mathbb{N}$. In what follows, denote by $M=\{1, \ldots, m\}$ the index set of all a priori unions in $\mathcal{P}$; for every $P$-game $\langle v, \mathcal{P}\rangle \in \mathcal{G}_{N}^{P}$ and every $k \in M$ let $v_{k}$ denote the subgame $\left.v\right|_{N_{k}}$; for every $i \in N$, let $k(i)$ be defined by the relation $i \in N_{k(i)}$; and for every $x \in \mathbb{R}^{N}$, let $x^{\mathcal{P}}=\left(x\left(N_{k}\right)\right)_{k \in M} \in \mathbb{R}^{M}$ stand for the vector of total payoffs to a priori unions.

One of the best-known values for games with coalition structure is the Owen value (Owen 1977) that can be seen as a two-step procedure in which the Shapley value applies twice. Namely, the Owen value assigns to player $i \in N$ his Shapley value in the game $\bar{v}_{k(i)}$, i.e.,

$$
O w_{i}(v, \mathcal{P})=S h_{i}\left(\bar{v}_{k(i)}\right), \quad \text { for all } i \in N
$$

while for every a priori union $N_{k}, k \in M$, the game $\bar{v}_{k} \in \mathcal{G}_{N_{k}}$ on the player set $N_{k}$ is given by

$$
\bar{v}_{k}(S)=S h_{k}\left(\hat{v}_{S}\right), \quad S \subseteq N_{k}
$$


where for every $S \subseteq N_{k}$ the game $\hat{v}_{S} \in \mathcal{G}_{M}$ on the player set $M$ of a priori unions is defined by

$$
\hat{v}_{S}(Q)=\left\{\begin{array}{ll}
v\left(\cup_{h \in Q} N_{h}\right), & k \notin Q, \\
v\left(\cup_{h \in Q \backslash\{k\}} N_{h} \cup S\right), & k \in Q,
\end{array} \quad \text { for all } Q \subseteq M\right.
$$

The Owen value is efficient, i.e., $\sum_{i \in N} O w_{i}(v, \mathcal{P})=v(N)$, and satisfies the quotient game property, i.e., for every a priori union the total payoff to the players within that union is determined by applying the Shapley value to the so-called quotient game being the game $v_{\mathcal{P}} \in \mathcal{G}_{M}$ in which the unions act as individual players,

$$
v_{\mathcal{P}}(Q)=v\left(\cup_{k \in Q} N_{k}\right), \text { for all } Q \subseteq M
$$

Notice that for every $k \in M$ the game $\hat{v}_{N_{k}}$ is equal to the quotient game $v_{\mathcal{P}}$.

Another value for games with coalition structure that also can be seen as a two-step procedure in which the Shapley value applies twice is the so-called two-step Shapley value $\psi$ introduced in Kamijo (2009). The two-step Shapley value first allocates to player $i \in N$ his Shapley value in the subgame on the a priori union $N_{k(i)}$ he belongs to and then distributes what remains of the Shapley value of its union in the quotient game equally among the union's members, i.e., for any $P$-game $\langle v, \mathcal{P}\rangle \in \mathcal{G}_{N}^{P}$,

$$
K a_{i}(v, \mathcal{P})=S h_{i}\left(v_{k(i)}\right)+\frac{S h_{k(i)}\left(v_{\mathcal{P}}\right)-v\left(N_{k(i)}\right)}{n_{k(i)}} .
$$

The Kamijo's two-step Shapley value is efficient and meets the quotient game property.

\subsection{Games with communication structure}

A communication structure on $N$ is specified by a graph $\Gamma$, undirected or directed, on $N$. A graph on $N$ consists of $N$ as the set of nodes and for an undirected graph a collection of unordered pairs $\Gamma \subseteq\{\{i, j\} \mid i, j \in N, i \neq j\}$ as the set of links between two nodes in $N$, and for a directed graph, or a digraph, a collection of ordered pairs $\Gamma \subseteq\{(i, j) \mid i, j \in N, i \neq j\}$ as the set of directed links from one node to another node in $N$. When it is necessary to specify the set of nodes $N$ in a graph $\Gamma$, we write $\Gamma_{N}$ instead of $\Gamma$. Let $\mathfrak{G}_{N}$ denote the set of all communication structures, undirected or directed, on $N$, and let $\mathcal{G}_{N}^{\Gamma}=\mathcal{G}_{N} \times \mathfrak{G}_{N}$. A pair $\langle v, \Gamma\rangle \in \mathcal{G}_{N}^{\Gamma}$ constitutes a game with graph (communication) structure, or simply a graph game, or a $\Gamma$-game, on $N$. A $\Gamma$-value is a mapping $\xi: \mathcal{G}_{N}^{\Gamma} \rightarrow \mathbb{R}^{N}$ that assigns to every $N \subset \mathbb{N}$ and every $\Gamma$-game $\langle v, \Gamma\rangle \in \mathcal{G}_{N}^{\Gamma}$ a payoff vector $\xi(v, \Gamma) \in \mathbb{R}^{N}$.

In a graph $\Gamma$ a sequence of different nodes $\left(i_{1}, \ldots, i_{r}\right), r \geq 2$, is a path in $\Gamma$ from node $i_{1}$ to node $i_{r}$ if for $h=1, \ldots, r-1$ it holds that $\left\{i_{h}, i_{h+1}\right\} \in \Gamma$ when $\Gamma$ is undirected and $\left\{\left(i_{h}, i_{h+1}\right),\left(i_{h+1}, i_{h}\right)\right\} \cap \Gamma \neq \emptyset$ when $\Gamma$ is directed. In a digraph $\Gamma$ a path $\left(i_{1}, \ldots, i_{r}\right)$ is a directed path from node $i_{1}$ to node $i_{r}$ if $\left(i_{h}, i_{h+1}\right) \in \Gamma$ for all $h=1, \ldots, r-1$. In a digraph $\Gamma, j \neq i$ is a successor of $i$ and $i$ is a predecessor of $j$ if there exists a directed path from $i$ to $j$. Given a digraph $\Gamma$ on $N$ and $i \in N$, the 
sets of predecessors and successors of $i$ in $\Gamma$ we denote correspondingly by $P^{\Gamma}(i)$ and $S^{\Gamma}(i)$; moreover, $\bar{P}^{\Gamma}(i)=P^{\Gamma}(i) \cup\{i\}$ and $\bar{S}^{\Gamma}(i)=S^{\Gamma}(i) \cup\{i\}$.

Given a graph $\Gamma$ on $N$, two nodes $i$ and $j$ in $N$ are connected if either there exists a path from node $i$ to node $j$, or $i$ and $j$ coincide. Graph $\Gamma$ on $N$ is connected if any two nodes in $N$ are connected. For a graph $\Gamma$ on $N$ and a coalition $S \subseteq N$, the subgraph of $\Gamma$ on $S$ is the graph $\left.\Gamma\right|_{S}=\{\{i, j\} \in \Gamma \mid i, j \in S\}$ on $S$ when $\Gamma$ is undirected and the digraph $\left.\Gamma\right|_{S}=\{(i, j) \in \Gamma \mid i, j \in S\}$ on $S$ when $\Gamma$ is directed. Given a graph $\Gamma$ on $N$, a coalition $S \subseteq N$ is connected if the subgraph $\left.\Gamma\right|_{S}$ is connected. For a graph $\Gamma$ on $N$ and coalition $S \subseteq N, C^{\Gamma}(S)$ is the set of all connected subcoalitions of $S, S / \Gamma$ is the set of maximal connected subcoalitions of $S$, called the components of $S$, and $(S / \Gamma)_{i}$ is the component of $S$ containing player $i \in S$. Notice that $S / \Gamma$ is a partition of $S$. For any $\langle v, \Gamma\rangle \in \mathcal{G}_{N}^{\Gamma}$, a payoff vector $x \in \mathbb{R}^{N}$ is component efficient if $x(C)=v(C)$, for every $C \in N / \Gamma$.

Following Myerson (1977), we assume that for $\Gamma$-games cooperation is possible only among connected players and concentrate on component efficient $\Gamma$-values. A $\Gamma$-value $\xi$ is component efficient $(\mathrm{CE})$ if for any $\langle v, \Gamma\rangle \in \mathcal{G}_{N}^{\Gamma}$, for all $C \in N / \Gamma$, $\sum_{i \in C} \xi_{i}(v, \Gamma)=v(C)$. Below for a $\Gamma$-game $\langle v, \Gamma\rangle \in \mathcal{G}_{N}^{\Gamma}$ we also consider the introduced in Myerson (1977) restricted game $v^{\Gamma} \in \mathcal{G}_{N}$ defined as

$$
v^{\Gamma}(S)=\sum_{C \in S / \Gamma} v(C), \quad \text { for all } S \subseteq N
$$

Hereinafter along with communication structures given by arbitrary undirected graphs we consider also those given by cycle-free undirected graphs and by directed graphs - linear graphs with linearly ordered players, rooted and sink forests. In an undirected graph $\Gamma$ a path $\left(i_{1}, \ldots, i_{r}\right), r \geq 3$, is a cycle in $\Gamma$ if $\left\{i_{r}, i_{1}\right\} \in \Gamma$. An undirected graph is cycle-free if it contains no cycles. A directed graph $\Gamma$ is a rooted tree if there is one node in $N$, called a root, having no predecessors in $\Gamma$ and there is a unique directed path in $\Gamma$ from this node to any other node in $N$. A directed graph $\Gamma$ is a sink tree if the directed graph composed by the same set of links as $\Gamma$ but with the opposite orientation is a rooted tree; in this case the root of a tree changes its meaning to the absorbing sink. A directed graph is a rooted/sink forest if it is composed by a number of disjoint rooted/sink trees. A linear graph is a directed graph that contains links only between subsequent nodes. Without loss of generality we may assume that in a linear graph nodes are ordered according to the natural order from 1 to $n$, i.e., linear graph $\Gamma \subseteq\{(i, i+1) \mid i=1, \ldots, n-1\}$.

For ease of notation given graph $\Gamma$ and link $\{i, j\} \in \Gamma$ if $\Gamma$ is undirected, or $(i, j) \in \Gamma$ if $\Gamma$ is directed, the subgraph $\Gamma \backslash\{\{i, j\}\}$, correspondingly $\Gamma \backslash\{(i, j)\}$, is denoted by $\left.\Gamma\right|_{-i j}$.

\subsection{Games with two-level communication structure}

We now consider situations in which the players are partitioned into a coalition structure $\mathcal{P}$ and are linked to each other by communication graphs. First, there is a communication graph $\Gamma_{M}$ on the set of a priori unions determined by the partition 
$\mathcal{P}$. Second, for each a priori union $N_{k}, k \in M$, there is a communication graph $\Gamma_{N_{k}}$ between the players in $N_{k}$. In what follows for simplicity of notation and when it causes no ambiguity we denote graphs $\Gamma_{N_{k}}$ within a priori unions $N_{k}, k \in M$, by $\Gamma_{k}$. Given a player set $N \subset \mathbb{N}$ and a coalition structure $\mathcal{P} \in \mathfrak{P}_{N}$, a two-level graph (communication) structure on $N$ is a tuple $\Gamma_{\mathcal{P}}=\left\langle\Gamma_{M},\left\{\Gamma_{k}\right\}_{k \in M}\right\rangle$. For every $N \subset \mathbb{N}$ and $\mathcal{P} \in \mathfrak{P}_{N}$ by $\mathfrak{G}_{N}^{\mathcal{P}}$ we denote the set of all two-level graph structures on $N$ with fixed $\mathcal{P}$. Let $\mathfrak{G}_{N}^{P}=\bigcup_{\mathcal{P} \in \mathfrak{P}_{N}} \mathfrak{G}_{N}^{\mathcal{P}}$ be the set of all two-level graph structures on $N$, and let $\mathcal{G}_{N}^{P \Gamma}=\mathcal{G}_{N} \times \mathfrak{G}_{N}^{P}$. A pair $\left\langle v, \Gamma_{\mathcal{P}}\right\rangle \in \mathcal{G}_{N}^{P \Gamma}$ constitutes a game with two-level graph (communication) structure, or simply a two-level graph game or a $P \Gamma$-game, on $N$. A $P \Gamma$-value is a mapping $\xi: \mathcal{G}_{N}^{P \Gamma} \rightarrow \mathbb{R}^{N}$ that assigns to every $N \subset \mathbb{N}$ and every $P \Gamma$-game $\left\langle v, \Gamma_{\mathcal{P}}\right\rangle \in \mathcal{G}_{N}^{P \Gamma}$ a payoff vector $\xi\left(v, \Gamma_{\mathcal{P}}\right) \in \mathbb{R}^{N}$.

Given a $P \Gamma$-game $\left\langle v, \Gamma_{\mathcal{P}}\right\rangle \in \mathcal{G}_{N}^{P \Gamma}$, one can consider the quotient $\Gamma$-game $\left\langle v_{\mathcal{P}}, \Gamma_{M}\right\rangle \in \mathcal{G}_{M}^{\Gamma}$ and the $\Gamma$-games within a priori unions $\left\langle v_{k}, \Gamma_{k}\right\rangle \in \mathcal{G}_{N_{k}}^{\Gamma}$ with $v_{k}=\left.v\right|_{N_{k}}, k \in M$, that model the bargaining between a priori unions for their total shares and the bargaining within each a priori union for the distribution of its total worth among the members taking also into account limited cooperation at both communication levels introduced by the communication graphs $\Gamma_{M}$ and $\Gamma_{k}, k \in M$. Moreover, given a $\Gamma$-value $\phi$, for any $\left\langle v, \Gamma_{\mathcal{P}}\right\rangle \in \mathcal{G}_{N}^{P \Gamma}$ with a graph structure $\Gamma_{M}$ on the level of a priori unions suitable for application of $\phi$ to the corresponding quotient $\Gamma$-game $\left\langle v_{\mathcal{P}}, \Gamma_{M}\right\rangle$, along with a subgame $v_{k}$ within a priori union $N_{k}, k \in M$, one can also consider a $\phi_{k}$-game $v_{k}^{\phi}$ defined as

$$
v_{k}^{\phi}(S)=\left\{\begin{array}{ll}
\phi_{k}\left(v_{\mathcal{P}}, \Gamma_{M}\right), & S=N_{k}, \\
v(S), & S \neq N_{k},
\end{array} \quad \text { for all } S \subseteq N_{k},\right.
$$

where $\phi_{k}\left(v_{\mathcal{P}}, \Gamma_{M}\right)$ is the payoff to $N_{k}$ given by $\phi$ in $\left\langle v_{\mathcal{P}}, \Gamma_{M}\right\rangle$. Then a $\Gamma$-game $\left\langle v_{k}^{\phi}, \Gamma_{k}\right\rangle \in \mathcal{G}_{N_{k}}^{\Gamma}$ models the bargaining within union $N_{k}$ for the distribution of its total share among the members taking into account restrictions on cooperation in $N_{k}$ given by $\Gamma_{k}$, when the share is obtained by the application of $\Gamma$-value $\phi$ at the upper level bargaining between a priori unions.

\section{Deletion link properties for two-level graph games}

As it is discussed in Khmelnitskaya (2014), a number of known component efficient $\Gamma$-values for games with undirected or directed communication structure such as for undirected graph games the Myerson value $\mu$ (cf., Myerson 1977) and the position value $\pi$ (cf., Meessen 1988; Borm et al. 1992; Slikker 2005) for arbitrary undirected graph games, the average tree solution AT (cf., Herings et al. 2008) and the compensation solution CS (cf., Béal et al. 2012) for undirected cycle-free graph games, or for directed graph games the upper equivalent solution UE, the lower equivalent solution LE and the equal loss solution EL for linear graph games (cf., van den Brink et al. 2007), the tree value $t$ for rooted forest and the sink value $s$ for sink forest digraph games (cf., Khmelnitskaya 2010), can be approached within the unified framework. Indeed, each one of these $\Gamma$-values is defined for $\Gamma$-games with suitable graph struc- 
ture and is characterized by two axioms, CE and one or another deletion link (DL) property (axiom), reflecting the relevant reaction of a $\Gamma$-value on the deletion of a link in the communication graph. The corresponding DL properties are fairness $(\mathrm{F})$, balanced link contributions (BLC), component fairness (CF), relative fairness (RF), upper equivalence (UE), lower equivalence (LE), equal loss property (EL), successor equivalence (SE), and predecessor equivalence (PE), and the characterization results are as follows:

$$
\begin{aligned}
\mathrm{CE}+\mathrm{F} \text { for all undirected } \Gamma \text {-games } & \Longleftrightarrow \mu(v, \Gamma), \\
\mathrm{CE}+\mathrm{BLC} \text { for all undirected } \Gamma \text {-games } & \Longleftrightarrow \pi(v, \Gamma), \\
\mathrm{CE}+\mathrm{CF} \text { for undirected cycle-free } \Gamma \text {-games } & \Longleftrightarrow A T(v, \Gamma), \\
\mathrm{CE}+\mathrm{RF} \text { for undirected cycle-free } \Gamma \text {-games } & \Longleftrightarrow C S(v, \Gamma), \\
\mathrm{CE}+\mathrm{UE} \text { for linear graph } \Gamma \text {-games } & \Longleftrightarrow U E(v, \Gamma), \\
\mathrm{CE}+\mathrm{LE} \text { for linear graph } \Gamma \text {-games } & \Longleftrightarrow L E(v, \Gamma), \\
\mathrm{CE}+\mathrm{EL} \text { for linear graph } \Gamma \text {-games } & \Longleftrightarrow E L(v, \Gamma), \\
\mathrm{CE}+\mathrm{SE} \text { for rooted forest } \Gamma \text {-games } & \Longleftrightarrow t(v, \Gamma), \\
\mathrm{CE}+\mathrm{PE} \text { for sink forest } \Gamma \text {-games } & \Longleftrightarrow s(v, \Gamma),
\end{aligned}
$$

This observation allows to identify each of the listed above $\Gamma$-values with the corresponding DL axiom. Given a DL axiom, let $\mathcal{G}_{N}^{D L} \subseteq \mathcal{G}_{N}^{\Gamma}$ denote a set of all $\langle v, \Gamma\rangle \in \mathcal{G}_{N}^{\Gamma}$ with $\Gamma$ suitable for DL application. Then

$$
\mathrm{CE}+\mathrm{DL} \text { on } \mathcal{G}_{N}^{D L} \Longleftrightarrow D L(v, \Gamma)
$$

Whence it simply follows that $F(v, \Gamma)=\mu(v, \Gamma)$ and $B L C(v, \Gamma)=\pi(v, \Gamma)$ for all undirected $\Gamma$-games, $C F(v, \Gamma)=A T(v, \Gamma)$ and $R F(v, \Gamma)=C S(v, \Gamma)$ for all undirected cycle-free $\Gamma$-games, $U E(v, \Gamma), L E(v, \Gamma)$, and $E L(v, \Gamma)$ are UE, LE, and EL solutions correspondingly for all linear graph $\Gamma$-games, $S E(v, \Gamma)=t(v, \Gamma)$ for all rooted forest $\Gamma$-games, and $P E(v, \Gamma)=s(v, \Gamma)$ for all sink forest $\Gamma$-games. Remark that all just discussed values are additive.

Next notice that every discussed DL axiom can be equivalently defined by an equality

$$
\Psi^{D L}\left(\xi(v, \Gamma), \Gamma^{\prime}\right)=0,
$$

where $\Psi^{D L}$ is an operator which for a $\Gamma$-value $\xi$ defined on $\mathcal{G}_{N}^{D L}$ and applied to a $\Gamma$-game $\langle v, \Gamma\rangle \in \mathcal{G}_{N}^{D L}$ assigns a real number representing the numerical evaluation of players' payoff reaction on the deletion of links in $\Gamma$ from a chosen set of links $\Gamma^{\prime} \subseteq \Gamma$ according to the considered DL axiom. For the above mentioned DL-axioms, we have $\Gamma^{\prime}=\Gamma$, and the corresponding operators of the DL-axioms are:

Fairness (F) For any player set $N \subset \mathbb{N}$, for every $\Gamma$-game $\langle v, \Gamma\rangle \in \mathcal{G}_{N}^{\Gamma}$,

$$
\Psi^{F}\left(\xi(v, \Gamma), \Gamma^{\prime}\right)=\sum_{i, j \in N \mid\{i, j\} \in \Gamma^{\prime}}\left|\left(\xi_{i}(v, \Gamma)-\xi_{i}\left(v,\left.\Gamma\right|_{-i j}\right)\right)-\left(\xi_{j}(v, \Gamma)-\xi_{j}\left(v,\left.\Gamma\right|_{-i j}\right)\right)\right|
$$


Balanced link contributions (BLC) For any player set $N \subset \mathbb{N}$, for every $\Gamma$-game $\langle v, \Gamma\rangle \in \mathcal{G}_{N}^{\Gamma}$,

$$
\begin{aligned}
\Psi^{B L C}\left(\xi(v, \Gamma), \Gamma^{\prime}\right)= & \sum_{i, j \in N} \mid \sum_{h \in N \mid\{i, h\} \in \Gamma^{\prime}}\left(\xi_{j}(v, \Gamma)-\xi_{j}(v, \Gamma \mid-i h)\right) \\
& -\sum_{h \in N \mid\{j, h\} \in \Gamma^{\prime}}\left(\xi_{i}(v, \Gamma)-\xi_{i}(v, \Gamma \mid-j h)\right) \mid,
\end{aligned}
$$

Component fairness (CF) For any player set $N \subset \mathbb{N}$, for every cycle-free $\Gamma$-game $\langle v, \Gamma\rangle \in \mathcal{G}_{N}^{\Gamma}$,

$$
\begin{aligned}
\Psi^{C F}\left(\xi(v, \Gamma), \Gamma^{\prime}\right)= & \sum_{i, j \in N \mid\{i, j\} \in \Gamma^{\prime}} \mid \frac{1}{\left|\left(N / \Gamma \mid{ }_{-i j}\right)_{i}\right|} \sum_{h \in(N / \Gamma \mid-i j)_{i}}\left(\xi_{h}(v, \Gamma)-\xi_{h}(v, \Gamma \mid-i j)\right) \\
& -\frac{1}{\left|\left(N /\left.\Gamma\right|_{-i j}\right)_{j}\right|} \sum_{h \in\left(N /\left.\Gamma\right|_{-i j}\right)_{j}}\left(\xi_{h}(v, \Gamma)-\xi_{h}\left(v,\left.\Gamma\right|_{-i j}\right)\right) \mid,
\end{aligned}
$$

Relative fairness (RF) For any player set $N \subset \mathbb{N}$, for every cycle-free $\Gamma$-game $\langle v, \Gamma\rangle \in \mathcal{G}_{N}^{\Gamma}$,

$$
\begin{aligned}
\Psi^{R F}\left(\xi(v, \Gamma), \Gamma^{\prime}\right)= & \sum_{i, j \in N \mid\{i, j\} \in \Gamma^{\prime}} \mid\left(\xi_{i}(v, \Gamma)-\frac{1}{\left|(N / \Gamma \mid-i j)_{i}\right|} \sum_{h \in\left(N /\left.\Gamma\right|_{-i j}\right)_{i}} \xi_{h}(v, \Gamma \mid-i j)\right) \\
& -\left(\xi_{j}(v, \Gamma)-\frac{1}{\left|\left(N /\left.\Gamma\right|_{-i j}\right)_{j}\right|} \sum_{h \in\left(N /\left.\Gamma\right|_{-i j}\right)_{j}} \xi_{h}\left(v,\left.\Gamma\right|_{-i j}\right)\right) \mid,
\end{aligned}
$$

Upper equivalence (UE) For any player set $N \subset \mathbb{N}$, for every linear graph $\Gamma$-game $\langle v, \Gamma\rangle \in \mathcal{G}_{N}^{\Gamma}$,

$$
\Psi^{U E}\left(\xi(v, \Gamma), \Gamma^{\prime}\right)=\sum_{i=1}^{n-1} \sum_{j=1}^{i}\left|\xi_{j}(v, \Gamma)-\xi_{j}\left(v,\left.\Gamma\right|_{-i, i+1}\right)\right|
$$

Lower equivalence (LE) For any player set $N \subset \mathbb{N}$, for every linear graph $\Gamma$-game $\langle v, \Gamma\rangle \in \mathcal{G}_{N}^{\Gamma}$,

$$
\Psi^{L E}\left(\xi(v, \Gamma), \Gamma^{\prime}\right)=\sum_{i=1}^{n-1} \sum_{j=i+1}^{n}\left|\xi_{j}(v, \Gamma)-\xi_{j}(v, \Gamma \mid-i, i+1)\right|
$$


Equal loss property (EL) For any player set $N \subset \mathbb{N}$, for every linear graph $\Gamma$-game $\langle v, \Gamma\rangle \in \mathcal{G}_{N}^{\Gamma}$,

$$
\begin{aligned}
& \Psi^{E L}\left(\xi(v, \Gamma), \Gamma^{\prime}\right) \\
& =\sum_{i=1}^{n-1}\left|\sum_{j=1}^{i}\left(\xi_{j}(v, \Gamma)-\xi_{j}\left(v,\left.\Gamma\right|_{-i, i+1}\right)\right)-\sum_{j=i+1}^{n}\left(\xi_{j}(v, \Gamma)-\xi_{j}\left(v,\left.\Gamma\right|_{-i, i+1}\right)\right)\right| .
\end{aligned}
$$

Successor equivalence (SE) For any player set $N \subset \mathbb{N}$, for every rooted forest $\Gamma$ game $\langle v, \Gamma\rangle \in \mathcal{G}_{N}^{\Gamma}$,

$$
\Psi^{S E}\left(\xi(v, \Gamma), \Gamma^{\prime}\right)=\sum_{i, j \in N \mid\{i, j\} \in \Gamma^{\prime}} \sum_{h \in \bar{S}^{\Gamma}(j)}\left|\xi_{h}(v, \Gamma)-\xi_{h}(v, \Gamma \mid-i j)\right| .
$$

Predecessor equivalence (PE) For any player set $N \subset \mathbb{N}$, for every sink forest $\Gamma$-game $\langle v, \Gamma\rangle \in \mathcal{G}_{N}^{\Gamma}$,

$$
\Psi^{P E}\left(\xi(v, \Gamma), \Gamma^{\prime}\right)=\sum_{i, j \in N \mid\{i, j\} \in \Gamma^{\prime}} \sum_{h \in \bar{P}^{\Gamma}(j)}\left|\xi_{h}(v, \Gamma)-\xi_{h}(v, \Gamma \mid-i j)\right| .
$$

The definition of deletion link axioms for $\Gamma$-values in terms of equality (3) allows to consider the corresponding deletion link axioms within a unified framework also for $P \Gamma$-values with respect to both communication levels, at the upper level with respect to the quotient $\Gamma$-game determining the total payoffs to a priori unions and at the lower level with respect to $\Gamma$-games within a priori unions determining the distribution of total payoffs within each a priori union. Let $\xi$ be a $P \Gamma$-value. By definition $\xi$ is a mapping $\xi: \mathcal{G}_{N}^{P \Gamma} \rightarrow \mathbb{R}^{N}$ that assigns a payoff vector to any $P \Gamma$-game on the player set $N \subset \mathbb{N}$. For every $N \subset \mathbb{N}$ a mapping $\xi=\left\{\xi_{i}\right\}_{i \in N}$ generates on the domain of $P \Gamma$-games on $N$ a mapping $\xi^{\mathcal{P}}: \mathcal{G}_{N}^{P \Gamma} \rightarrow \mathbb{R}^{M}, \xi^{\mathcal{P}}=\left\{\xi_{k}^{\mathcal{P}}\right\}_{k \in M}$, with $\xi_{k}^{\mathcal{P}}=\sum_{i \in N_{k}} \xi_{i}$, $k \in M$, that assigns to every $P \Gamma$-game on $N$ a vector of total payoffs to all a priori unions, and $m$ mappings $\xi^{k}: \mathcal{G}_{N}^{P \Gamma} \rightarrow \mathbb{R}^{N_{k}}, \xi^{k}=\left\{\xi_{i}\right\}_{i \in N_{k}}, k \in M$, assigning payoffs to players within a priori unions. For a given $(m+1)$-tuple of deletion link axioms $\left\langle\mathrm{DL}^{\mathcal{P}},\left\{\mathrm{DL}^{k}\right\}_{k \in M}\right\rangle$ for $\Gamma$-values, let $\mathcal{G}_{N}^{D L^{\mathcal{P}},\left\{D L^{k}\right\}_{k \in M}} \subseteq \mathcal{G}_{N}^{P \Gamma}$ be the set of $P \Gamma$-games composed of $P \Gamma$-games $\left\langle v, \Gamma_{\mathcal{P}}\right\rangle$ with graph structures $\Gamma_{\mathcal{P}}=\left\langle\Gamma_{M},\left\{\Gamma_{k}\right\}_{k \in M}\right\rangle$ such that $\left\langle v_{\mathcal{P}}, \Gamma_{M}\right\rangle \in \mathcal{G}_{M}^{D L^{\mathcal{P}}}$ and $\left\langle v_{k}^{D L^{\mathcal{P}}}, \Gamma_{k}\right\rangle \in \mathcal{G}_{N_{k}}^{D L^{k}}, k \in M$. Given a $(m+1)$-tuple of deletion link axioms $\left\langle\mathrm{DL}^{\mathcal{P}},\left\{\mathrm{DL}^{k}\right\}_{k \in M}\right\rangle$ for $\Gamma$-values and a $P \Gamma$-game $\left\langle v, \Gamma_{\mathcal{P}}\right\rangle \in$ $\mathcal{G}_{N}^{D L^{\mathcal{P}},\left\{D L^{k}\right\}_{k \in M}}$, we may consider the operators (4)-(12) applied to the $\Gamma$-value $\xi^{\mathcal{P}}$ assigning the total payoffs to a priori unions to evaluate its reaction on deletion of links in graph $\Gamma_{M}$, or applied to the $\Gamma$-values $\xi^{k}, k \in M$, assigning payoffs to single players within a priori unions to evaluate their reaction on deletion of links in the corresponding graphs $\Gamma_{k}$. Based on the last observation, we define axioms of Quotient DL property and Union DL property for $P \Gamma$-values defined on $\mathcal{G}_{N}^{D L^{\mathcal{P}},\left\{D L^{k}\right\}_{k \in M}}$ as follows: 
Quotient DL property (QDL) For any player set $N \subset \mathbb{N}$ and $P \Gamma$-game $\left\langle v, \Gamma_{\mathcal{P}}\right\rangle \in$ $\mathcal{G}_{N}^{D L^{\mathcal{P}},\left\{D L^{k}\right\}_{k \in M}}, \Gamma_{\mathcal{P}}=\left\langle\Gamma_{M},\left\{\Gamma_{h}\right\}_{h \in M}\right\rangle$,

$$
\Psi^{D L^{\mathcal{P}}}\left(\xi^{\mathcal{P}}\left(v, \Gamma_{\mathcal{P}}\right), \Gamma_{M}\right)=0
$$

where $\Psi^{D L^{\mathcal{P}}}$ is given by one of the operators (4)-(12) and in the corresponding formulas $\left.\Gamma_{\mathcal{P}}\right|_{-k l}=\left\langle\left.\Gamma_{M}\right|_{-k l},\left\{\Gamma_{h}\right\}_{h \in M}\right\rangle$ for every link $\{k, l\} \in \Gamma_{M}$ if $\Gamma_{M}$ is undirected, or $(k, l) \in \Gamma_{M}$ if $\Gamma_{M}$ is directed.

Union DL property (UDL) For any player set $N \subset \mathbb{N}, P \Gamma$-game $\left\langle v, \Gamma_{\mathcal{P}}\right\rangle \in$ $\mathcal{G}_{N}^{D L^{\mathcal{P}},\left\{D L^{k}\right\}_{k \in M}}, \Gamma_{\mathcal{P}}=\left\langle\Gamma_{M},\left\{\Gamma_{h}\right\}_{h \in M}\right\rangle$, and $k \in M$,

$$
\Psi^{D L^{k}}\left(\xi^{k}\left(v, \Gamma_{\mathcal{P}}\right), \Gamma_{k}\right)=0
$$

where $\Psi^{D L^{k}}$ is given by one of the operators (4)-(12) and in the corresponding formulas $\left.\Gamma_{\mathcal{P}}\right|_{-i j}=\left.\Gamma_{\mathcal{P}}\right|_{-i j} ^{k}=\left\langle\Gamma_{M},\left\{\widehat{\Gamma}_{h}\right\}_{h \in M}\right\rangle$ with $\widehat{\Gamma}_{h}=\Gamma_{h}$ for $h \neq k$ and $\widehat{\Gamma}_{k}=\left.\Gamma_{k}\right|_{-i j}$ for every link $\{i, j\} \in \Gamma_{k}$ if $\Gamma_{k}$ is undirected, or $(i, j) \in \Gamma_{k}$ if $\Gamma_{k}$ is directed.

For example, if we consider the Myerson fairness (F) as a DL axiom, QDL and $\mathrm{UDL}^{k}$ can be denoted QF and $\mathrm{UF}^{k}$ correspondingly. In fact in this case QF coincides with quotient fairness $(\mathrm{QF})$ and $m$-tuple of axioms $\left(\mathrm{UF}^{1}, \ldots, \mathrm{UF}^{m}\right)$ coincides with union fairness (UF) employed in van den Brink et al. (2016).

QDL and UDL axioms for $P \Gamma$-values provide the uniform approach to various deletion link properties on both bargaining levels. This allows to introduce within the unified framework two families of $P \Gamma$-values based on the adaptation of the two-step distribution procedures of Owen and Kamijo respectively for games with coalition structure to the case when the cooperation between and within a priori unions is restricted by communication graphs and when different combinations of known component efficient solution concepts on both communication levels could be applied. Moreover, this allows to include into consideration not only combinations of undirected communication graphs but also combinations including some types of digraphs.

\section{Kamijo-type values for two-level graph games}

In this section we consider the family of $P \Gamma$-values based on the adaptation of the Kamijo's two-step distribution procedure for $P$-games. We introduce these values axiomatically by means of six axioms among which QDL and UDL as defined in the previous section. The other four axioms are defined below. To begin with, Quotient component efficiency requires that each component on the upper level between a priori unions distributes fully its total worth among the players of the a priori unions forming this component. 
Quotient component efficiency (QCE) For any $N \subset \mathbb{N}$, any $\left\langle v, \Gamma_{\mathcal{P}}\right\rangle \in \mathcal{G}_{N}^{P \Gamma}, \Gamma_{\mathcal{P}}=$ $\left\langle\Gamma_{M},\left\{\Gamma_{k}\right\}_{k \in M}\right\rangle$,

$$
\sum_{k \in K} \sum_{i \in N_{k}} \xi_{i}\left(v, \Gamma_{\mathcal{P}}\right)=v_{\mathcal{P}}(K), \quad \text { for all } K \in M / \Gamma_{M}
$$

The second axiom is a straightforward adaptation of the standard covariance under strategic equivalence to the case of $P \Gamma$-games. Two games $v, w \in \mathcal{G}_{N}$ are strategically equivalent if there are $a \in \mathbb{R}_{++}$and $b \in \mathbb{R}^{n}$ such that

$$
w(S)=a v(S)+b(S), \quad \text { for all } S \subseteq N
$$

Covariance under strategic equivalence (COV) For any player set $N \subset \mathbb{N}$ and $P \Gamma$-game $\left\langle v, \Gamma_{\mathcal{P}}\right\rangle \in \mathcal{G}_{N}^{P \Gamma}, \Gamma_{\mathcal{P}}=\left\langle\Gamma_{M},\left\{\Gamma_{k}\right\}_{k \in M}\right\rangle$, for any $a \in \mathbb{R}_{++}$and $b \in \mathbb{R}^{n}$ it holds that

$$
\xi\left(a v+b, \Gamma_{\mathcal{P}}\right)=a \xi\left(v, \Gamma_{\mathcal{P}}\right)+b,
$$

where the game $(a v+b) \in \mathcal{G}_{N}$ is defined by $(a v+b)(S)=a v(S)+b(S)$ for all $S \subseteq N$.

The next axiom requires equal payoffs to all members of every a priori union $N_{k}$, $k \in M$, for which all subcoalitions $S \subseteq N_{k}$ with nonzero worth $v_{k}(S) \neq 0$ are disconnected. In this case every connected coalition possesses zero worth, i.e., communication between the members of every connected coalition is useless, and so, the asymmetries among the players created by game $v_{k}$ and by their locations in graph $\Gamma_{k}$ on $N_{k}$ vanish. Therefore, it makes sense to treat all players of $N_{k}$ symmetrically. Remark that the condition that $v(S) \neq 0$ implies $S$ to be disconnected is equivalent to $v_{k}^{\Gamma_{k}} \equiv \mathbf{0}$, i.e., the axiom requires that all players in $N_{k}$ obtain the same payoffs if the Myerson restricted game $v_{k}^{\Gamma_{k}}$ is a null game. The latter observation determines the name of the axiom.

Union null restricted game property (UNRGP) For any player set $N \subset \mathbb{N}$ and $P \Gamma$-game $\left\langle v, \Gamma_{\mathcal{P}}\right\rangle \in \mathcal{G}_{N}^{P \Gamma}, \Gamma_{\mathcal{P}}=\left\langle\Gamma_{M},\left\{\Gamma_{k}\right\}_{k \in M}\right\rangle$, if for some $k \in M$, for all $S \subseteq N_{k}$, $v_{k}(S) \neq 0$ implies $S \notin C^{\Gamma_{k}}\left(N_{k}\right)$, then it holds that for all $i, j \in N_{k}, i \neq j$,

$$
\xi_{i}\left(v, \Gamma_{\mathcal{P}}\right)=\xi_{j}\left(v, \Gamma_{\mathcal{P}}\right)
$$

The last axiom determines the distribution of the total shares obtained by internally disconnected a priori unions at the upper level bargaining between a priori unions among the components of these unions. Imagine that each a priori union $N_{k}, k \in M$, is a public institution (e.g. university, hospital, or firm) of which every component $C \in N_{k} / \Gamma_{k}$ is an independent unit (e.g. the faculties within a university, medical departments within a hospital, or production plants within a firm). First public institutions $N_{k}, k \in M$ compete among themselves for their annual budgets from the government. Once obtained the budget, institution $N_{k}$ has to decide how much to give 
to each of its independent units. At this stage the independent units of an institution compete against each other for the best possible shares from the institution's budget. Similarly as in the competition among the public institutions, the total payoff to a unit depends on the total productivity of each of the units, but not on the productivity of the smaller collaborating teams within the units. Our last axiom requires the total payoff to a component of any a priori union to be independent of the so-called internal coalitions, each of which is a proper subcoalition of some component of one of the given a priori unions, or more precisely, a coalition $\emptyset \neq S \subseteq N$ is internal if there is $k \in M$ such that $S \subset C$ for some $C \in N_{k} / \Gamma_{k}$. From now on, given a player set $N \subset \mathbb{N}$, a partition $\mathcal{P}=\left\{N_{1}, \ldots, N_{m}\right\}$ of $N$, and a set of communication graphs $\left\{\Gamma_{k}\right\}_{k \in M}$ on a priori unions $N_{k}, k \in M$, the set of all internal coalitions we denote by $\operatorname{Int}\left(N, \mathcal{P},\left\{\Gamma_{k}\right\}_{k \in M}\right)$. It is worth to remark that, of course, the worths of internal coalitions play a crucial role in the redistribution of the total payoff obtained by a component among its members, but the axiom does not concern this.

Union component payoff independence of internal coalitions (UCPIIC) For any player set $N \subset \mathbb{N}$ and two $P \Gamma$-games $\left\langle v, \Gamma_{\mathcal{P}}\right\rangle,\left\langle w, \Gamma_{\mathcal{P}}\right\rangle \in \mathcal{G}_{N}^{P \Gamma}$ with the same $\Gamma_{\mathcal{P}}=$ $\left\langle\Gamma_{M},\left\{\Gamma_{k}\right\}_{k \in M}\right\rangle$ and such that $w(S)=v(S)$ for all $S \subseteq N, S \notin \operatorname{Int}\left(N, \mathcal{P},\left\{\Gamma_{k}\right\}_{k \in M}\right)$, it holds that for every $k \in M$, for all $C \in N_{k} / \Gamma_{k}$,

$$
\sum_{i \in C} \xi_{i}\left(v, \Gamma_{\mathcal{P}}\right)=\sum_{i \in C} \xi_{i}\left(w, \Gamma_{\mathcal{P}}\right)
$$

Our first theorem provides an axiomatic characterization of the a family of $P \Gamma$ values.

Theorem 1 For any $(m+1)$-tuple of deletion link axioms $\left\langle\mathrm{DL}^{\mathcal{P}},\left\{\mathrm{DL}^{k}\right\}_{k \in M}\right\rangle$ such that $\mathrm{DL}^{\mathcal{P}}$ is not $R F$ and the set of $\mathrm{DL}^{k}, k \in M$, axioms is restricted to $F$ and $C F$, there is a unique $P \Gamma$-value defined on $\mathcal{G}_{N}^{D L^{\mathcal{P}},\left\{D L^{k}\right\}_{k \in M}}$ that meets axioms $Q C E, Q D L, U D L$, COV, UNRGP, and UCPIIC, and for every $P \Gamma$-game $\left\langle v, \Gamma_{\mathcal{P}}\right\rangle \in \mathcal{G}_{N}^{D L^{\mathcal{P}},\left\{D L^{k}\right\}_{k \in M}}$ it is given by

$\xi_{i}\left(v, \Gamma_{\mathcal{P}}\right)=D L_{i}^{k(i)}\left(v_{k(i)}, \Gamma_{k(i)}\right)+\frac{D L_{k(i)}^{\mathcal{P}}\left(v_{\mathcal{P}}, \Gamma_{M}\right)-v_{k(i)}^{\Gamma_{k(i)}}\left(N_{k(i)}\right)}{n_{k(i)}}$, for all $i \in N$

Before proving Theorem 1, we formulate some remarks.

Remark 1 It is not difficult to trace a relation between the $P \Gamma$-value $\xi$ given by (16) and the two-step Shapley value (1). Indeed, for a $P \Gamma$-game $\langle v, \Gamma \mathcal{P}\rangle \in \mathcal{G}_{N}^{F^{\mathcal{P}},\left\{F^{k}\right\}_{k \in M}}$, $\xi\left(v, \Gamma_{\mathcal{P}}\right)=K a\left(v^{\Gamma_{\mathcal{P}}}, \mathcal{P}\right)$, where $v^{\Gamma_{\mathcal{P}}} \in \mathcal{G}_{N}$ is determined as

$$
v^{\Gamma_{\mathcal{P}}}(S)=\left\{\begin{array}{ll}
v_{\mathcal{P}}^{\Gamma_{M}}(Q), & Q \subseteq M: S=\cup_{q \in Q} N_{q}, \\
v_{k}^{\Gamma_{k}}(S), & S \subseteq N_{k}, k \in M, \\
0, & \text { otherwise },
\end{array} \quad \text { for all } S \subseteq N\right.
$$


i.e., when $S$ is the union of number of a priori unions its worth is defined according to the Myerson restricted quotient game, when $S$ is a subset of some a priori union its worth is defined according to the Myerson restricted game within the union, otherwise the worth of $S$ is zero.

Because of the mentioned similarity, from now on we refer to the $P \Gamma$-value (16) as to the Kamijo-type $\left\langle D L^{\mathcal{P}},\left\{D L^{k}\right\}_{k \in M}\right\rangle$-value, denoted further by $K a^{\left\langle D L^{\mathcal{P}},\left\{D L^{k}\right\}_{k \in M}\right\rangle}$.

Remark 2 Axiom RF cannot be used at any level in Theorem 1. The reason is that it gives rise to the compensation solution at the level where RF is applied and the compensation solution does not satisfy COV on its domain. Furthermore, the $P \Gamma$ value $K a^{\left\langle D L^{\mathcal{P}},\left\{D L^{k}\right\}_{k \in M}\right\rangle}$ violates the UDL property if in the $(m+1)$-tuple of deletion link axioms $\left\langle\mathrm{DL}^{\mathcal{P}},\left\{\mathrm{DL}^{k}\right\}_{k \in M}\right\rangle$ among the axioms $\mathrm{DL}^{k}, k \in M$ there are axioms BLC, UE, LE, EL, SE, and PE. The reason is that the second summand in the numerator of the second term in the right-hand side of (16) is sensitive to the deletion of different links in graph $\Gamma_{k}$, and therefore, for these cases the equality $\Psi^{D L^{k}}\left(\xi^{k}\left(v, \Gamma_{\mathcal{P}}\right), \Gamma_{k}\right)=$ $\Psi^{D L^{k}}\left(D L^{k}\left(v_{k}, \Gamma_{k}\right), \Gamma_{k}\right)=0$ in general does not hold.

Proof (Theorem 1) I. [EXISTENCE]. We show that under the hypothesis of the theorem the $P \Gamma$-value $\xi=K a^{\left\langle D L^{\mathcal{P}},\left\{D L^{k}\right\}_{k \in M}\right\rangle}$ defined on $\mathcal{G}_{N}^{D L^{\mathcal{P}},\left\{D L^{k}\right\}_{k \in M}}$ by (16) meets the axioms QCE, QDL, UDL, COV, UNRGP and UCPIIC. Consider an arbitrary $P \Gamma$ game $\left\langle v, \Gamma_{\mathcal{P}}\right\rangle \in \mathcal{G}_{N}^{D L^{\mathcal{P}},\left\{D L^{k}\right\}_{k \in M}}$.

QCE By the definition (16) of $\xi$ and component efficiency of each $D L^{k}$-value for all $k \in M$ it holds that

$$
\sum_{i \in N_{k}} \xi_{i}\left(v, \Gamma_{\mathcal{P}}\right)=D L_{k}^{\mathcal{P}}\left(v_{\mathcal{P}}, \Gamma_{M}\right)
$$

Thus, for any $K \in M / \Gamma_{M}$ we have

$$
\sum_{k \in K} \sum_{i \in N_{k}} \xi_{i}\left(v, \Gamma_{\mathcal{P}}\right)=\sum_{k \in K} D L_{k}^{\mathcal{P}}\left(v_{\mathcal{P}}, \Gamma_{M}\right)=v_{\mathcal{P}}(K),
$$

where the second equality follows from component efficiency of $D L^{\mathcal{P}}$-value.

QDL From (17) we obtain that $\xi^{\mathcal{P}}\left(v, \Gamma_{\mathcal{P}}\right)=D L^{\mathcal{P}}\left(v_{\mathcal{P}}, \Gamma_{M}\right)$. Whence it follows that

$$
\Psi^{D L^{\mathcal{P}}}\left(\xi^{\mathcal{P}}\left(v, \Gamma_{\mathcal{P}}\right), \Gamma_{M}\right)=\Psi^{D L^{\mathcal{P}}}\left(D L^{\mathcal{P}}\left(v_{\mathcal{P}}, \Gamma_{M}\right), \Gamma_{M}\right)=0
$$

where the last equality holds true since the $D L^{\mathcal{P}}$-value for $\Gamma$-games meets $D L^{\mathcal{P}}$.

UDL We need to show that if the set of axioms $\mathrm{DL}^{k}, k \in M$, is restricted to $\mathrm{F}$ and $\mathrm{CF}$, then for all $k \in M, \Psi^{D L^{k}}\left(\xi^{k}\left(v, \Gamma_{\mathcal{P}}\right), \Gamma_{k}\right)=0$.

Let for some $k \in M, \mathrm{DL}^{k}=\mathrm{F}$. Then since by definition for any link $\{i, j\} \in \Gamma_{k}$, $\left.\Gamma_{\mathcal{P}}\right|_{-i j}=\left.\Gamma_{\mathcal{P}}\right|_{-i j} ^{k}=\left\langle\Gamma_{M},\left\{\widehat{\Gamma}_{h}\right\}_{h \in M}\right\rangle$ with $\widehat{\Gamma}_{h}=\Gamma_{h}$ for $h \neq k$, and $\widehat{\Gamma}_{k}=\left.\Gamma_{k}\right|_{-i j}$, we 
obtain that

$$
\begin{aligned}
& \Psi^{F}\left(\xi^{k}\left(v, \Gamma_{\mathcal{P}}\right), \Gamma_{k}\right) \\
& \stackrel{(4)}{=} \sum_{i, j \in N_{k} \mid\{i, j\} \in \Gamma_{k}}\left|\left(\xi_{i}^{k}\left(v, \Gamma_{\mathcal{P}}\right)-\xi_{i}^{k}\left(v,\left.\Gamma_{\mathcal{P}}\right|_{-i j}\right)\right)-\left(\xi_{j}^{k}\left(v, \Gamma_{\mathcal{P}}\right)-\xi_{j}^{k}\left(v,\left.\Gamma_{\mathcal{P}}\right|_{-i j}\right)\right)\right| \\
& \stackrel{(16)}{=} \sum_{i, j \in N_{k} \mid\{i, j\} \in \Gamma_{k}} \mid\left(F_{i}^{k}\left(v_{k}, \Gamma_{k}\right)+\frac{D L_{k}^{\mathcal{P}}\left(v_{\mathcal{P}}, \Gamma_{M}\right)-v_{k}^{\Gamma_{k}}\left(N_{k}\right)}{n_{k}}\right. \\
& \quad-F_{i}^{k}\left(v_{k},\left.\Gamma_{k}\right|_{-i j}\right)+\frac{D L_{k}^{\mathcal{P}}\left(v_{\mathcal{P}}, \Gamma_{M}\right)-v_{k}^{\left.\Gamma_{k}\right|_{-i j}}\left(N_{k}\right)}{\left.n_{k}\right)} \\
& \quad-\left(F_{j}^{k}\left(v_{k}, \Gamma_{k}\right)+\frac{D L_{k}^{\mathcal{P}}\left(v_{\mathcal{P}}, \Gamma_{M}\right)-v_{k}^{\Gamma_{k}}\left(N_{k}\right)}{n_{k}}-F_{j}^{k}\left(v_{k},\left.\Gamma_{k}\right|_{-i j}\right)\right. \\
& \left.\quad+\frac{D L_{k}^{\mathcal{P}}\left(v_{\mathcal{P}}, \Gamma_{M}\right)-v_{k}^{\left.\Gamma_{k}\right|_{-i j}}\left(N_{k}\right)}{n_{k}}\right) \mid \\
& \quad \sum_{i, j \in N_{k} \mid\{i, j\} \in \Gamma_{k}}\left|\left(F_{i}\left(v_{k}, \Gamma_{k}\right)-F_{i}\left(v_{k},\left.\Gamma_{k}\right|_{-i j}\right)\right)-\left(F_{j}\left(v_{k}, \Gamma_{k}\right)-F_{j}\left(v_{k},\left.\Gamma_{k}\right|_{-i j}\right)\right)\right| \\
& \stackrel{(4)}{=} \Psi_{\left(F\left(v_{k}, \Gamma_{k}\right), \Gamma_{k}\right)=0,}
\end{aligned}
$$

where the last equality holds true since the $F$-value for $\Gamma$-games (the Myerson value) meets fairness F. Using the similar arguments as above we prove that for all $k \in M$ for which $\mathrm{DL}^{k}=\mathrm{CF}, \Psi^{C F}\left(\xi^{k}\left(v, \Gamma_{\mathcal{P}}\right), \Gamma_{k}\right)=0$.

COV Pick any $a \in \mathbb{R}_{++}$and $b \in \mathbb{R}^{n}$. Then, for all $i \in N$,

$$
\begin{aligned}
\xi_{i}\left(a v+b, \Gamma_{\mathcal{P}}\right) \stackrel{(16),(2)}{=} & D L_{i}^{k(i)}\left((a v+b)_{k(i)}, \Gamma_{k(i)}\right) \\
& +\frac{D L_{k(i)}^{\mathcal{P}}\left((a v+b)_{\mathcal{P}}, \Gamma_{M}\right)-\sum_{C \in N_{k(i)} / \Gamma_{k(i)}}(a v+b)(C)}{n_{k(i)}} \\
= & a D L_{i}^{k(i)}\left(v_{k(i)}, \Gamma_{k(i)}\right)+b_{i} \\
& +\frac{a D L_{k(i)}^{\mathcal{P}}\left(v_{\mathcal{P}}, \Gamma_{M}\right)+b\left(N_{k(i)}\right)-\sum_{C \in N_{k(i)} / \Gamma_{k(i)}}(a v(C)+b(C))}{n_{k(i)}} \\
= & a \xi_{i}\left(v, \Gamma_{\mathcal{P}}\right)+b_{i},
\end{aligned}
$$

where the second equality is true because each of the considered $D L^{k}$-values, $k \in M$, and $D L^{\mathcal{P}}$-values meets COV on its domain, and $(a v+b)_{\mathcal{P}}(Q)=a v_{\mathcal{P}}(Q)+b\left(\cup_{k \in Q} N_{k}\right)$ for all $Q \subseteq M$; and the third equality is due to the equality $\sum_{C \in N_{k(i)} / \Gamma_{k(i)}} b(C)=$ $b\left(N_{k(i)}\right)$, since $N_{k(i)} / \Gamma_{k(i)}$ forms a partition of $N_{k(i)}$.

UNRGP Assume that for the chosen $P \Gamma$-game $\left\langle v, \Gamma_{\mathcal{P}}\right\rangle$ there exists $k \in M$ such that for all $S \subseteq N_{k}, v_{k}(S) \neq 0$ implies $S \notin C^{\Gamma_{k}}\left(N_{k}\right)$. Every of the considered $D L^{k}$-values, 
the Myerson value and the average tree solution, are determined only by worths of connected coalitions, and therefore, $D L_{i}^{k}\left(v_{k}, \Gamma_{k}\right)=0$ for all $i \in N_{k}$. Moreover, as it was already mentioned earlier, the above assumption is equivalent to $v_{k}^{\Gamma_{k}} \equiv \mathbf{0}$, which implies that $v(C)=0$ for every $C \in N_{k} / \Gamma_{k}$. Hence, from (16) it follows that for all $i \in N_{k}$,

$$
\xi_{i}\left(v, \Gamma_{\mathcal{P}}\right)=\frac{D L_{k}^{\mathcal{P}}\left(v_{\mathcal{P}}, \Gamma_{M}\right)}{n_{k}}
$$

where the right side is independent of $i$, from which it follows that $P \Gamma$-value $\xi$ meets UNRGP.

UCPIIC. Take any $k \in M$ and $C \in N_{k} / \Gamma_{k}$. The component efficiency of each of the considered $D L^{k}$-values implies $D L^{k}\left(v_{k}, \Gamma_{k}\right)(C)=v(C)$. Then from (16) it follows that

$$
\sum_{i \in C} \xi\left(v, \Gamma_{\mathcal{P}}\right)=v(C)+\frac{c}{n_{k}}\left(D L_{k}^{\mathcal{P}}\left(v_{\mathcal{P}}, \Gamma_{M}\right)-v^{\Gamma_{k}}\left(N_{k}\right)\right)
$$

where the right side is independent of worths of internal coalitions, i.e. $P \Gamma$-value $\xi$ meets UCPIIC.

II. [UNIQUENESS]. Assume that a $(m+1)$-tuple of deletion link axioms $\left\langle\mathrm{DL}^{\mathcal{P}}\right.$, $\left.\left\{\mathrm{DL}^{k}\right\}_{k \in M}\right\rangle$ such that the set of axioms $\mathrm{DL}^{k}, k \in M$, is restricted to $\mathrm{F}$ and $\mathrm{CF}$, is given. We show that there exists at most one $P \Gamma$-value on $\mathcal{G}_{N}^{D L^{\mathcal{P}},\left\{D L^{k}\right\}_{k \in M}}$ that satisfies axioms QCE, QDL, UDL, COV, UNRGP, and UCPIIC. Let $\phi$ be such $P \Gamma$-value on $\mathcal{G}_{N}^{D L^{\mathcal{P}},\left\{D L^{k}\right\}_{k \in M}}$. Take an arbitrary $P \Gamma$-game $\left\langle v, \Gamma_{\mathcal{P}}\right\rangle \in \mathcal{G}_{N}^{D L^{\mathcal{P}},\left\{D L^{k}\right\}_{k \in M}}$. Fix some $k \in M$. We start by determining the union payoffs $\phi_{k}^{\mathcal{P}}\left(v, \Gamma_{\mathcal{P}}\right), k \in M$, by induction on the number of links in $\Gamma_{M}$ similarly as it is done in the proof of uniqueness of the Myerson value for $\Gamma$-games, cf., Myerson (1977).

INITIALIZATION: If $\left|\Gamma_{M}\right|=0$ then for all $k \in M$ the set of neighboring unions $\{h \in M \mid\{h, k\} \in \Gamma\}=\emptyset$, and therefore by QCE and definition of the quotient game $v_{\mathcal{P}}, \phi_{k}^{\mathcal{P}}\left(v, \Gamma_{\mathcal{P}}\right)=v_{\mathcal{P}}(\{k\})=v\left(N_{k}\right)$.

INDUCTION HYPOTHESIS: Assume that the values $\phi_{k}^{\mathcal{P}}\left(v, \Gamma_{\mathcal{P}}^{\prime}\right), k \in M$, have been determined for all two-level graph structures $\Gamma_{\mathcal{P}}^{\prime}=\left\langle\Gamma^{\prime},\left\{\Gamma_{h}\right\}_{h \in M}\right\rangle$ with $\Gamma^{\prime}$ such that $\left|\Gamma^{\prime}\right|<\left|\Gamma_{M}\right|$.

INDUCTION STEP: Let $Q \in M / \Gamma_{M}$ be a component in graph $\Gamma_{M}$ on $M$. If $Q \subseteq M$ is a singleton, let $Q=\{k\}$, then from $\mathrm{QCE}$ it follows that $\phi_{k}^{\mathcal{P}}\left(v, \Gamma_{\mathcal{P}}\right)=v\left(N_{k}\right)$. If $q \geq 2$, then there exists a spanning tree $\left.\widetilde{\Gamma} \subseteq \Gamma_{M}\right|_{Q}$ on $Q$ with the number of links $|\widetilde{\Gamma}|=q-1$. By QDL it holds that

$$
\Psi^{D L^{\mathcal{P}}}\left(\phi^{\mathcal{P}}\left(v, \Gamma_{\mathcal{P}}\right), \Gamma_{M}\right)=0
$$

The above equality in fact provides for each link $\{k, l\} \in \Gamma_{M}$ some equality relating values of $\phi_{h}^{\mathcal{P}}\left(v, \Gamma_{\mathcal{P}}\right), h \in M$, with values of distinct $\phi_{h}^{\mathcal{P}}\left(v,\left.\Gamma_{\mathcal{P}}\right|_{-k l}\right), h \in M$. Since 
$\left|\Gamma_{M}\right|-k l|=| \Gamma_{M} \mid-1$, from the induction hypothesis it follows that for all links $\{k, l\} \in \Gamma_{M}\left((k, l) \in \Gamma_{M}\right)$ the payoffs $\phi_{h}^{\mathcal{P}}\left(v,\left.\Gamma_{\mathcal{P}}\right|_{-k l}\right), h \in M$, are already determined. Thus with respect to $q-1$ links $\{k, l\} \in \widetilde{\Gamma}$, (18) yields $q-1$ linearly independent linear equations in the $q$ unknown payoffs $\phi_{k}^{\mathcal{P}}\left(v, \Gamma_{\mathcal{P}}\right), k \in Q$. Moreover, by QCE it holds that

$$
\sum_{k \in Q} \phi_{k}^{\mathcal{P}}\left(v, \Gamma_{\mathcal{P}}\right)=v_{\mathcal{P}}(Q)
$$

All these $q$ equations are linearly independent. Whence it follows that for every $Q \in$ $M / \Gamma$, all payoffs $\phi_{k}^{\mathcal{P}}\left(v, \Gamma_{\mathcal{P}}\right), k \in Q$, are uniquely determined. Notice that in the proof of the induction step, every possible spanning tree $\widetilde{\Gamma}$ yields the same solution for the values $\phi_{k}^{\mathcal{P}}\left(v, \Gamma_{\mathcal{P}}\right), k \in Q$, because otherwise a solution does not exist, which contradicts the already proved "existence" part of the proof of the theorem.

Next, we show that the individual payoffs $\phi_{i}\left(v, \Gamma_{\mathcal{P}}\right), i \in N_{k}$, are uniquely determined. This part of the proof is also by induction, now on the number of links in $\Gamma_{k}$.

INITIALIZATION: Assume that $\left|\Gamma_{k}\right|=0$. Let $v_{0}$ be the 0 -normalization of the TU game $v$, i.e., $v_{0}(S)=v(S)-\sum_{i \in S} v(\{i\})$ for all $S \subseteq N$, and let $\left(v_{0}\right)_{k}=\left.v_{0}\right|_{N_{k}}$. As already shown above, the union payoffs $\phi_{k}^{\mathcal{P}}\left(w, \Gamma_{\mathcal{P}}\right), k \in M$, are uniquely determined for any $P \Gamma$-game $\left\langle w, \Gamma_{\mathcal{P}}\right\rangle \in \mathcal{G}_{N}^{D L^{\mathcal{P}},\left\{D L^{k}\right\}_{k \in M}}$. In particular, the "union payoffs" $\phi_{k}^{\mathcal{P}}\left(v_{0}, \Gamma_{\mathcal{P}}\right), k \in M$, are uniquely determined. By definition $\left(v_{0}\right)_{k}(\{i\})=0$ for every $i \in N_{k}$, and therefore, $\left(v_{0}\right)_{k}^{\Gamma_{k}} \equiv \mathbf{0}$ since $\left|\Gamma_{k}\right|=0$. Then, from UNRGP it follows that

$$
\phi_{i}\left(v_{0}, \Gamma_{\mathcal{P}}\right)=\frac{\phi_{k}^{\mathcal{P}}\left(v_{0}, \Gamma_{\mathcal{P}}\right)}{n_{k}}, \quad \text { for all } i \in N_{k}
$$

Whence by COV we obtain

$$
\phi_{i}\left(v, \Gamma_{\mathcal{P}}\right)=v(\{i\})+\frac{\phi_{k}^{\mathcal{P}}\left(v^{0}, \Gamma_{\mathcal{P}}\right)}{n_{k}}, \quad \text { for all } i \in N_{k},
$$

i.e., for every $i \in N_{k}, \phi_{i}\left(v, \Gamma_{\mathcal{P}}\right)$ is uniquely determined.

INDUCTION HYPOTHESIS: Let $\Gamma_{\mathcal{P}}^{\prime}$ denote the two-level graph structure $\left\langle\Gamma_{M}\right.$, $\left.\left\{\Gamma_{h}^{\prime}\right\}_{h \in M}\right\rangle$ with $\Gamma_{h}^{\prime}=\Gamma_{h}$ if $h \neq k$ and $\Gamma_{k}^{\prime}=\Gamma^{\prime}$ for some graph $\Gamma^{\prime}$ on $N_{k}$. Assume that the values $\phi_{i}\left(v, \Gamma_{\mathcal{P}}^{\prime}\right)$ have been determined for every $\Gamma^{\prime}$ with $\left|\Gamma^{\prime}\right|<\left|\Gamma_{k}\right|$.

InDUCTION STEP: For every $S \in \operatorname{Int}\left(N, \mathcal{P},\left\{\Gamma_{k}\right\}_{k \in M}\right)$ let $C_{S} \in N_{k} / \Gamma_{k}$ be the unique component such that $S \subset C_{S}$. Consider a game $w \in \mathcal{G}_{N}$ defined as

$$
w(S)=\left\{\begin{array}{ll}
v(S), & S \notin \operatorname{Int}\left(N, \mathcal{P},\left\{\Gamma_{k}\right\}_{k \in M}\right), \\
\frac{s v\left(C_{S}\right)}{c_{S}}, & S \in \operatorname{Int}\left(N, \mathcal{P},\left\{\Gamma_{k}\right\}_{k \in M}\right),
\end{array} \quad \text { for all } S \subseteq N\right.
$$

For the 0-normalization $w_{0}$ of $w,\left(w_{0}\right)_{k}=\left(w_{k}\right)_{0}$. The subgame $w_{k}$ is an additive game and, therefore, $\left(w_{k}\right)_{0} \equiv \mathbf{0}$. Then, due to UNRGP, similar as in the Initialization step, it follows that 


$$
\phi_{i}\left(w_{0}, \Gamma_{\mathcal{P}}\right)=\frac{\phi_{k}^{\mathcal{P}}\left(w_{0}, \Gamma_{\mathcal{P}}\right)}{n_{k}}, \quad \text { for all } i \in N_{k}
$$

Whence by COV we obtain

$$
\phi_{i}\left(w, \Gamma_{\mathcal{P}}\right)=w(\{i\})+\frac{\phi_{k}^{\mathcal{P}}\left(w_{0}, \Gamma_{\mathcal{P}}\right)}{n_{k}}, \quad \text { for all } i \in N_{k}
$$

Consider a component $C \in N_{k} / \Gamma_{k}$. From the above equality it follows that

$$
\sum_{i \in C} \phi_{i}\left(w, \Gamma_{\mathcal{P}}\right)=\sum_{i \in C} w(\{i\})+\frac{c}{n_{k}} \phi_{k}^{\mathcal{P}}\left(w_{0}, \Gamma_{\mathcal{P}}\right) \stackrel{(19)}{=} v(C)+\frac{c}{n_{k}} \phi_{k}^{\mathcal{P}}\left(w_{0}, \Gamma_{\mathcal{P}}\right)
$$

By the definition (19) of $w, w(S)=v(S)$ for all $S \subseteq N, S \notin \operatorname{Int}\left(N, \mathcal{P},\left\{\Gamma_{k}\right\}_{k \in M}\right)$. Whence by UCPIIC it follows that

$$
\sum_{i \in C} \phi_{i}\left(v, \Gamma_{\mathcal{P}}\right)=v(C)+\frac{c}{n_{k}} \mathrm{DL}_{k}^{\mathcal{P}}\left(w_{0, \mathcal{P}}, \Gamma_{M}\right), \quad \text { for all } C \in N_{k} / \Gamma_{k} .
$$

Next, if $c=1$, then $C$ is a singleton and the payoff $\phi_{i}\left(v, \Gamma_{\mathcal{P}}\right)$ of the only player $i \in C$ is uniquely determined by (20). If $c \geq 2$, then there exists a spanning tree $\left.\widetilde{\Gamma} \subseteq \Gamma_{k}\right|_{C}$ on $C$ with the number of links $|\widetilde{\Gamma}|=c-1$. By UDL it holds that

$$
\Psi^{D L^{k}}\left(\phi^{k}\left(v, \Gamma_{\mathcal{P}}\right), \Gamma_{k}\right)=0
$$

The above equality in fact provides for any link $\{i, j\} \in \Gamma_{k}$ some equality relating values of $\phi_{h}\left(v, \Gamma_{\mathcal{P}}\right), h \in N_{k}$, with values of distinct $\phi_{h}\left(v,\left.\Gamma_{\mathcal{P}}\right|_{-i j} ^{k}\right)$. Since $\left|\Gamma_{k}\right|_{-i j} \mid=$ $\left|\Gamma_{k}\right|-1$, by the induction hypothesis it follows that for all links $\{i, j\} \in \Gamma_{k}$ the payoffs $\phi_{h}\left(v,\left.\Gamma_{\mathcal{P}}\right|_{-i j} ^{k}\right), h \in N_{k}$, are already determined. Thus with respect to $c-1$ links $\{i, j\} \in \widetilde{\Gamma},(21)$ yields $c-1$ linearly independent linear equations in the $c$ unknown payoffs $\phi_{i}\left(v, \Gamma_{\mathcal{P}}\right), i \in C$. These $c-1$ equations together with (20) form a system of $c$ linearly independent equations in the $c$ unknown payoffs $\phi_{i}\left(v, \Gamma_{\mathcal{P}}\right)$, $i \in C$. Hence, for every $C \in N_{k} / \Gamma_{k}$, all payoffs $\phi_{i}\left(v, \Gamma_{\mathcal{P}}\right), i \in C$, are uniquely determined.

\section{Logical independence of the axioms}

Given a $(m+1)$-tuple of deletion link axioms $\left\langle\mathrm{DL}^{\mathcal{P}},\left\{\mathrm{DL}^{k}\right\}_{k \in M}\right\rangle$, such that the set of $\mathrm{DL}^{k}, k \in M$, axioms is restricted to $\mathrm{F}, \mathrm{CF}$, and $\mathrm{RF}$, the logical independence of the axioms in Theorem 1 is demonstrated by the following examples of $P \Gamma$-values:

- The $P \Gamma$-value $\xi^{(1)}$ assigning in every $\left\langle v, \Gamma_{\mathcal{P}}\right\rangle \in \mathcal{G}_{N}^{\mathrm{DL}^{\mathcal{P}},\left\{\mathrm{DL}^{k}\right\}_{k \in M}}$ to every player $i \in N$ a payoff

$$
\xi_{i}^{(1)}\left(v, \Gamma_{\mathcal{P}}\right)=\frac{D L_{k(i)}^{\mathcal{P}}\left(v_{\mathcal{P}}, \Gamma_{M}\right)}{n_{k(i)}}
$$


satisfies all axioms except COV.

- The $P \Gamma$-value $\xi^{(2)}$ assigning in every $\left\langle v, \Gamma_{\mathcal{P}}\right\rangle \in \mathcal{G}_{N}^{\mathrm{DL}^{\mathcal{P}},\left\{\mathrm{DL}^{k}\right\}_{k \in M}}$ to every player $i \in N$ a payoff

$$
\xi_{i}^{(2)}\left(v, \Gamma_{\mathcal{P}}\right)=v(\{i\})+\frac{D L_{k(i)}^{\mathcal{P}}\left(v_{\mathcal{P}}, \Gamma_{M}\right)-\sum_{j \in N_{k(i)}} v(\{j\})}{n_{k(i)}}
$$

satisfies every axiom except UCPIIC.

- The $P \Gamma$-value $\xi^{(3)}$ assigning in every $\left\langle v, \Gamma_{\mathcal{P}}\right\rangle \in \mathcal{G}_{N}^{\mathrm{DL}^{\mathcal{P}},\left\{\mathrm{DL}^{k}\right\}_{k \in M}}$ to every player $i \in N$ a payoff

$$
\xi_{i}^{(3)}\left(v, \Gamma_{\mathcal{P}}\right)=D L_{i}^{k(i)}\left(v_{k(i)}, \Gamma_{k(i)}\right)+\frac{S h_{k(i)}\left(v_{\left(M / \Gamma_{M}\right)_{k(i)}}\right)-v^{\Gamma_{k(i)}}\left(N_{k}\right)}{n_{k(i)}}
$$

satisfies every axiom except QDL.

- The $P \Gamma$-value $\xi^{(4)}$ assigning in every $\left\langle v, \Gamma_{\mathcal{P}}\right\rangle \in \mathcal{G}_{N}^{\mathrm{DL}^{\mathcal{P}},\left\{\mathrm{DL}^{k}\right\}_{k \in M}}$ to every player $i \in N$ a payoff

$$
\xi_{i}^{(4)}\left(v, \Gamma_{\mathcal{P}}\right)=E L_{i}^{k(i)}\left(v_{k(i)}, \Gamma_{k(i)}\right)+\frac{D L_{k(i)}^{\mathcal{P}}\left(v_{\mathcal{P}}, \Gamma_{M}\right)-v^{\Gamma_{k(i)}}\left(N_{k}\right)}{n_{k(i)}}
$$

satisfies every axiom except UDL.

- The $P \Gamma$-value $\xi^{(5)}$ assigning in every $\left\langle v, \Gamma_{\mathcal{P}}\right\rangle \in \mathcal{G}_{N}^{\mathrm{DL}^{\mathcal{P}},\left\{\mathrm{DL}^{k}\right\}_{k \in M}}$ to every player $i \in N$ a payoff

$$
\xi_{i}^{(5)}\left(v, \Gamma_{\mathcal{P}}\right)=D L_{i}^{k(i)}\left(v_{k(i)}, \Gamma_{k(i)}\right)+\frac{v\left(N_{k(i)}\right)-v^{\Gamma_{k(i)}}\left(N_{k(i)}\right)}{n_{k(i)}}
$$

satisfies every axiom except QCE.

- The $P \Gamma$-value $\xi^{(6)}$ assigning in every $\left\langle v, \Gamma_{\mathcal{P}}\right\rangle \in \mathcal{G}_{N}^{\mathrm{DL}^{\mathcal{P}},\left\{\mathrm{DL}^{k}\right\}_{k \in M}}$ to every player $i \in N$ a payoff

$$
\xi_{i}^{(6)}\left(v, \Gamma_{\mathcal{P}}\right)=K a_{i}^{\left\langle D L^{\mathcal{P}},\left\{D L^{k}\right\}_{k \in M}\right\rangle}\left(v, \Gamma_{\mathcal{P}}\right)+a_{i}-\frac{1}{n_{k(i)}} \sum_{j \in N_{k(i)}} a_{j},
$$

where $\left(a_{j}\right)_{j \in N} \in \mathbb{R}^{n}$ is any vector of real numbers such that not all coordinates are equal, satisfies every axiom except UNRGP.

\section{Owen-type values for two-level graph games}

In this section we consider another family of $P \Gamma$-values based on the adaptation of the Owen's two-step distribution procedure for $P$-games. We introduce these values axiomatically by means of four axioms. The first three axioms are QCE, QDL, and 
UDL already employed in the previous section for characterization of the Kamijotype $\left\langle D L^{\mathcal{P}},\left\{D L^{k}\right\}_{k \in M}\right\rangle$-values. The fourth axiom of fair distribution of the surplus within unions was first introduced in van den Brink et al. (2016) where it was used for the axiomatization of the Owen-type value for $P \Gamma$-games which is based on the application of the Myerson value on both communication levels. This axiom requires balanced average payoff variation for all components within a priori union in case the other components leave the game.

Fair distribution of the surplus within unions (FDSU) For any $N \subset \mathbb{N}$, any $\left\langle v, \Gamma_{\mathcal{P}}\right\rangle \in \mathcal{G}_{N}^{P \Gamma}, \Gamma_{\mathcal{P}}=\left\langle\Gamma_{M},\left\{\Gamma_{k}\right\}_{k \in M}\right\rangle, k \in M$, any $C, C^{\prime} \in N_{k} / \Gamma_{k}$,

$$
\frac{1}{c} \sum_{i \in C}\left(\xi_{i}\left(v, \Gamma_{\mathcal{P}}\right)-\xi_{i}\left(v_{C}^{k}, \Gamma_{\mathcal{P}_{C}^{k}}\right)\right)=\frac{1}{c^{\prime}} \sum_{i \in C^{\prime}}\left(\xi_{i}\left(v, \Gamma_{\mathcal{P}}\right)-\xi_{i}\left(v_{C^{\prime}}^{k}, \Gamma_{\mathcal{P}_{C^{\prime}}^{k}}\right)\right),
$$

where for $k \in M$ and component $C \in N_{k} / \Gamma_{k}, v_{C}^{k}$ denotes the subgame $\left.v\right|_{\left(N \backslash N_{k}\right) \cup C}$ of $v$ with respect to the coalition $\left(N \backslash N_{k}\right) \cup C, \mathcal{P}_{C}^{k}$ denotes the partition on $\left(N \backslash N_{k}\right) \cup C$ consisting of union $C$ and all unions $N_{h}$ in $\mathcal{P}, h \neq k$, and $\Gamma_{\mathcal{P}_{C}^{k}}=\left\langle\Gamma_{M},\left\{\widetilde{\Gamma}_{h}\right\}_{h \in M}\right\rangle$ with $\widetilde{\Gamma}_{k}=\left.\Gamma_{k}\right|_{C}$ and $\widetilde{\Gamma}_{h}=\Gamma_{h}$ for all $h \in M \backslash\{k\}$, denotes the two-level communication structure that is obtained from $\left\langle\Gamma_{M},\left\{\Gamma_{h}\right\}_{h \in M}\right\rangle$ by replacing the communication graph $\Gamma_{k}$ by its restriction on $C \subseteq N_{k}$.

The next theorem extends the Owen-type value for $P \Gamma$-games studied in van den Brink et al. (2016) by allowing the application of different combinations of known component efficient solution concepts for $\Gamma$-games on both communication levels.

Theorem 2 For any $(m+1)$-tuple of deletion link axioms $\left\langle\mathrm{DL}^{\mathcal{P}},\left\{\mathrm{DL}^{k}\right\}_{k \in M}\right\rangle$ such that the set of $\mathrm{DL}^{k}, k \in M$, axioms is restricted to $F, C F$, and $R F$, there is a unique $P \Gamma$-value defined on $\mathcal{G}_{N}^{D L^{\mathcal{P}},\left\{D L^{k}\right\}_{k \in M}}$ that meets axioms $Q C E, F D S U, Q D L$, and UDL. For every $P \Gamma$-game $\langle v, \Gamma \mathcal{P}\rangle \in \mathcal{G}_{N}^{D L^{\mathcal{P}},\left\{D L^{k}\right\}_{k \in M}}$ it is given by

$\xi_{i}\left(v, \Gamma_{\mathcal{P}}\right)=D L_{i}^{k(i)}\left(\tilde{v}_{k(i)}, \Gamma_{k(i)}\right)+\frac{D L_{k(i)}^{\mathcal{P}}\left(v_{\mathcal{P}}, \Gamma_{M}\right)-\sum_{C \in N_{k(i)} / \Gamma_{k(i)}} \tilde{v}_{k(i)}(C)}{n_{k(i)}}, \quad i \in N$,

where for all $k \in M, \tilde{v}_{k} \in \mathcal{G}_{N_{k}}$ is defined as

$$
\tilde{v}_{k}(S)=D L_{k}^{\mathcal{P}}\left(\hat{v}_{S}, \Gamma_{M}\right), \quad \text { for all } S \subseteq N_{k},
$$

and for every $S \subseteq N_{k}, \hat{v}_{S} \in \mathcal{G}_{M}$ is given by

$$
\hat{v}_{S}(Q)=\left\{\begin{array}{ll}
v\left(\cup_{h \in Q} N_{h}\right), & k \notin Q, \\
v\left(\cup_{h \in Q \backslash\{k\}} N_{h} \cup S\right), & k \in Q,
\end{array} \text { for all } Q \subseteq M .\right.
$$

The proof strategy is similar to that applied in van den Brink et al. (2016), a careful reader may find the proof in "Appendix". We skip the proof of logical independence of axioms since it can be easily obtained by modification of the examples used for the 
proof in van den Brink et al. (2016) in case of $\left\langle\mathrm{DL}^{\mathcal{P}},\left\{\mathrm{DL}^{k}\right\}_{k \in M}\right\rangle=\left\langle\mathrm{F}^{\mathcal{P}},\left\{\mathrm{F}^{k}\right\}_{k \in M}\right\rangle$, i.e., when the Myerson fairness $\mathrm{F}$ is applied on both levels.

Note that in case when $\left\langle\mathrm{DL}^{\mathcal{P}},\left\{\mathrm{DL}^{k}\right\}_{k \in M}\right\rangle=\left\langle\mathrm{F}^{\mathcal{P}},\left\{\mathrm{F}^{k}\right\}_{k \in M}\right\rangle$, the statement of Theorem 2 coincides with the statement of both Proposition 4.1 and Theorem 4.2 proved in van den Brink et al. (2016) together and the $P \Gamma$-value $\xi$ given by (23) coincides as well with the Owen-type $P \Gamma$-value introduced there. Because of the latter coincidence, from now on we refer to the $P \Gamma$-value (23) as to the Owen-type $\left\langle D L^{\mathcal{P}},\left\{D L^{k}\right\}_{k \in M}\right\rangle$-value denoted further by $O w^{\left\langle D L^{\mathcal{P}},\left\{D L^{k}\right\}_{k \in M}\right\rangle}$.

Observe also that the Owen-type $\left\langle D L^{\mathcal{P}},\left\{D L^{k}\right\}_{k \in M}\right\rangle$-value admits the similar twostep construction procedure as the Owen value for games with coalition structure. The difference is that instead of two applications of the Shapley value used in the case of the classical Owen value, in the Owen-type $\left\langle D L^{\mathcal{P}},\left\{D L^{k}\right\}_{k \in M}\right\rangle$-value different known component efficient $\Gamma$-values can be applied on both communication levels.

Remark 3 Note that different from Theorem 1, in Theorem 2 RF can be used at any level due to the fact that COV employed in Theorem 1 is replaced by FDSU in Theorem 2, and the latter axiom is compatible with the compensation solution characterized by RF. By the reasons similar to those mentioned in Remark 2, the $P \Gamma$-value $O w^{\left\langle D L^{\mathcal{P}},\left\{D L^{k}\right\}_{k \in M}\right\rangle}$ violates the UDL property when there are axioms BLC, UE, LE, EL, SE, and PE among the axioms $\mathrm{DL}^{k}, k \in M$ in the $(m+1)$-tuple $\left\langle\mathrm{DL}^{\mathcal{P}},\left\{\mathrm{DL}^{k}\right\}_{k \in M}\right\rangle$.

\section{Conclusion}

We conclude by a final comparison of the two families of values introduced by Theorems 1 and 2. Observe that all considered $P \Gamma$-values meet QCE, QDL, UDL, and COV (the last one only if RF is not involved). However, while the Owentype $\left\langle D L^{\mathcal{P}},\left\{D L^{k}\right\}_{k \in M}\right\rangle$-values satisfy FDSU, but violate UNRGP and UCPIIC, the Kamijo-type $\left\langle D L^{\mathcal{P}},\left\{D L^{k}\right\}_{k \in M}\right\rangle$-values vice versa satisfy UNRGP and UCPIIC, but violate FDSU. The summary of the properties is given in the following table, where the axioms needed for our axiomatizations are marked by $*$.

\begin{tabular}{llllllll}
\hline Value & QCE & QDL & UDL & COV & FDSU & UNRGP & UCPIIC \\
\hline $\begin{array}{l}\text { Owen-type } \\
\left\langle D L^{\mathcal{P}},\left\{D L^{k}\right\}_{k \in M}\right\rangle \text {-value }\end{array}$ & $+^{*}$ & $+^{*}$ & $+^{*}$ & + & $+^{*}$ & - & - \\
$\begin{array}{l}\text { Kamijo-type } \\
\left\langle D L^{\mathcal{P}},\left\{D L^{k}\right\}_{k \in M}\right\rangle \text {-value }\end{array}$ & $+^{*}$ & $+^{*}$ & $+^{*}$ & $+^{*}$ & - & $+^{*}$ & $+^{*}$ \\
\hline
\end{tabular}

Open Access This article is distributed under the terms of the Creative Commons Attribution 4.0 International License (http://creativecommons.org/licenses/by/4.0/), which permits unrestricted use, distribution, and reproduction in any medium, provided you give appropriate credit to the original author(s) and the source, provide a link to the Creative Commons license, and indicate if changes were made. 


\section{Appendix}

Proof (Theorem 2) I. [EXISTENCE]. We show that under the hypothesis of the theorem the $P \Gamma$-value $\xi=O w^{\left\langle D L^{\mathcal{P}},\left\{D L^{k}\right\}_{k \in M}\right\rangle}$ defined on $\mathcal{G}_{N}^{D L^{\mathcal{P}},\left\{D L^{k}\right\}_{k \in M}}$ by (23) meets the axioms QCE, QDL, FDSU, and UDL. Consider an arbitrary $P \Gamma$-game $\left\langle v, \Gamma_{\mathcal{P}}\right\rangle \in$ $\mathcal{G}_{N}^{D L^{\mathcal{P}},\left\{D L^{k}\right\}_{k \in M}}$.

QCE, QDL, UDL The proof of the fact that the Owen-type $\left\langle D L^{\mathcal{P}},\left\{D L^{k}\right\}_{k \in M}\right\rangle$-value meets these axioms is similar to the proof of same statement for the Kamijo-type $\left\langle D L^{\mathcal{P}},\left\{D L^{k}\right\}_{k \in M}\right\rangle$-value in Theorem 1, and so we skip it.

FDSU From (23) we obtain that for every $C \in N_{k} / \Gamma_{k}$ it holds that

$$
\begin{aligned}
\sum_{i \in C} \xi_{i}\left(v, \Gamma_{\mathcal{P}}\right) & \stackrel{(23)}{=} \sum_{i \in C} D L_{i}^{k}\left(\tilde{v}_{k}, \Gamma_{k}\right)+\frac{c}{n_{k}}\left(D L_{k}^{\mathcal{P}}\left(v_{\mathcal{P}}, \Gamma_{M}\right)-\sum_{H \in N_{k} / \Gamma_{k}} \tilde{v}_{k}(H)\right) \\
& =\tilde{v}_{k}(C)+\frac{c}{n_{k}}\left(D L_{k}^{\mathcal{P}}\left(v_{\mathcal{P}}, \Gamma_{M}\right)-\sum_{H \in N_{k} / \Gamma_{k}} \tilde{v}_{k}(H)\right),
\end{aligned}
$$

where the second equality is due to component efficiency of $D L^{k}$-value for $\Gamma$-games. Further,

$$
\begin{aligned}
\sum_{i \in C} \xi_{i}\left(v_{C}^{k}, \Gamma_{\mathcal{P}_{C}^{k}}\right) \stackrel{(23)}{=} & \left.\sum_{i \in C} D L_{i}^{k} \widetilde{\left(\left(v_{C}^{k}\right)_{k}\right.},\left.\Gamma_{k}\right|_{C}\right) \\
& +\frac{c}{n_{k}}\left(D L_{k}^{\mathcal{P}}\left(\left(v_{C}^{k}\right)_{\mathcal{P}_{C}^{k}}, \Gamma_{M}\right)-\widetilde{\sum_{H \in C /\left.\Gamma_{k}\right|_{C}}} \widetilde{\left(v_{C}^{k}\right)_{k}}(H)\right) \\
= & \widetilde{\left(v_{C}^{k}\right)_{k}}(C)+\widetilde{\frac{c}{n_{k}}}\left(D L_{k}^{\mathcal{P}}\left(\left(v_{C}^{k}\right)_{\mathcal{P}_{C}^{k}}, \Gamma_{M}\right)-\widetilde{\left(v_{C}^{k}\right)_{k}}(C)\right)=\tilde{v}_{k}(S),
\end{aligned}
$$

where the second equality is due to component efficiency of $D L^{k}$-value for $\Gamma$ games and $C$ being the only component in $\left.\Gamma_{k}\right|_{C}$, and the third equality follows from equality $\widetilde{\left(v_{C}^{k}\right)_{k}}(C)=\tilde{v}_{k}(C)$ which holds true because $\hat{v}_{C}=\widehat{\left(v_{C}^{k}\right)}$, and equalities $D L_{k}^{\mathcal{P}}\left(\left(v_{C}^{k}\right)_{\mathcal{P}_{C}^{k}}, \Gamma_{M}\right)=D L_{k}^{\mathcal{P}}\left(\hat{v}_{C}, \Gamma_{M}\right) \stackrel{\text { def }}{=} \tilde{v}_{k}(C)$ first of which holds true because $\left(v_{C}^{k}\right)_{\mathcal{P}_{C}^{k}}=\widehat{\left(v_{C}^{k}\right)_{C}}=\hat{v}_{C}$. Thus,

$$
\frac{1}{c} \sum_{i \in C}\left(\xi_{i}\left(v, \Gamma_{\mathcal{P}}\right)-\xi_{i}\left(v_{C}^{k}, \Gamma_{\mathcal{P}_{C}^{k}}\right)\right)=\frac{1}{n_{k}}\left(D L_{k}^{\mathcal{P}}\left(v_{\mathcal{P}}, \Gamma_{M}\right)-\sum_{H \in N_{k} / \Gamma_{k}} \tilde{v}_{k}(H)\right)
$$

where the right side of the latter equality is independent of $C$. 
II. [UNIQUENESS]. Assume that a $(m+1)$-tuple of deletion link axioms $\left\langle\mathrm{DL}^{\mathcal{P}}\right.$, $\left.\left\{\mathrm{DL}^{k}\right\}_{k \in M}\right\rangle$ such that the set of axioms $\mathrm{DL}^{k}, k \in M$, is restricted to $\mathrm{F}, \mathrm{CF}$, and $\mathrm{RF}$, is given. We show that there exists at most one $P \Gamma$-value on $\mathcal{G}_{N}^{D L^{\mathcal{P}},\left\{D L^{k}\right\}_{k \in M}}$ that satisfies axioms QCE, FDSU, QDL, and UDL. Let $\phi$ be such $P \Gamma$-value on $\mathcal{G}_{N}^{D L^{\mathcal{P}},\left\{D L^{k}\right\}_{k \in M}}$. Given an arbitrary $P \Gamma$-game $\left\langle v, \Gamma_{\mathcal{P}}\right\rangle \in \mathcal{G}_{N}^{D L^{\mathcal{P}},\left\{D L^{k}\right\}_{k \in M}}$ we show that the individual payoffs $\phi_{i}\left(v, \Gamma_{\mathcal{P}}\right), i \in N$, are uniquely determined.

Similar to the proof of Theorem 1 we may show that the union payoffs $\phi_{k}^{\mathcal{P}}\left(v, \Gamma_{\mathcal{P}}\right)$, $k \in M$, are uniquely determined. Applying the same strategy, for every $k \in M$ and any subset $C \subseteq N_{k}$ we determine the union payoffs $\phi_{k}^{\mathcal{P}}\left(v_{C}^{k}, \Gamma_{\mathcal{P}_{C}^{k}}\right)$ in the game $\left(v_{C}, \Gamma_{\mathcal{P}_{C}^{k}}\right)$, in which $v_{C}^{k}$ denotes the subgame $\left.v\right|_{\left(N \backslash N_{k}\right) \cup C}$ of $v$ with respect to the coalition $\left(N \backslash N_{k}\right) \cup C$, and $\Gamma_{\mathcal{P}_{C}^{k}}$ denotes the two-level communication structure $\left\langle\Gamma_{M},\left\{\Gamma_{h}\right\}_{h \in M}\right\rangle$, where $\Gamma_{M}$ is the communication graph on the partition $\left(\mathcal{P} \backslash\left\{N_{k}\right\}\right) \cup\{C\}$ and the communication graph $\Gamma_{k}$ is replaced by its restriction on $C$. Notice that now for $k \in M$, the union payoff $\phi_{k}^{\mathcal{P}}\left(v_{C}^{k}, \Gamma_{\mathcal{P}_{C}^{k}}\right)$ is the total payoff to the players in $C$ in the game $\left(v_{C}^{k}, \Gamma_{\mathcal{P}_{C}^{k}}\right)$.

Next we determine the individual payoffs in each a priori union $N_{k}, k \in M$. For this first we show that for each component $C \in N_{k} / \Gamma_{k}$ the total payoff to the players in $C$ is uniquely determined. The payoff $\phi_{k}^{\mathcal{P}}\left(v, \Gamma_{\mathcal{P}}\right)$ to the a priori union $N_{k}$ has been already determined, so

$$
\sum_{i \in N_{k}} \phi_{i}\left(v, \Gamma_{\mathcal{P}}\right)=\phi_{k}^{\mathcal{P}}\left(v, \Gamma_{\mathcal{P}}\right)
$$

If $N_{k}$ is the unique component in $N_{k} / \Gamma_{k}$, then FDSU does not state any requirement. When $N_{k} / \Gamma_{k}$ consists of multiple components, then for every component $C \in N_{k} / \Gamma_{k}$, from FDSU it follows that

$$
\frac{\sum_{i \in C} \phi_{i}\left(v, \Gamma_{\mathcal{P}}\right)-\phi_{k}^{\mathcal{P}}\left(v_{C}^{k}, \Gamma_{\mathcal{P}_{C}^{k}}\right)}{c}=\frac{\phi_{k}^{\mathcal{P}}\left(v, \Gamma_{\mathcal{P}}\right)-\sum_{K \in N_{k} / \Gamma_{k}} \phi_{k}^{\mathcal{P}}\left(v_{K}^{k}, \Gamma_{\mathcal{P}_{K}^{k}}\right)}{n_{k}} .
$$

Notice that every payoff $\phi_{k}^{\mathcal{P}}$ in this equation has already been determined, and therefore, $\sum_{i \in C} \phi_{i}\left(v, \Gamma_{\mathcal{P}}\right)$ is uniquely determined.

The rest of the proof we proceed by induction similar to the proof of Theorem 1. Take some $k \in M$. Let $\Gamma_{\mathcal{P}}^{\prime}$ denote the two-level graph structure $\left\langle\Gamma_{M},\left\{\Gamma_{h}^{\prime}\right\}_{h \in M}\right\rangle$ with $\Gamma_{h}^{\prime}=\Gamma_{h}$ if $h \neq k$ and $\Gamma_{k}^{\prime}=\Gamma^{\prime}$ for some graph $\Gamma^{\prime}$ on $N_{k}$.

InitializATION: If $\left|\Gamma_{k}\right|=0$ then $\{i\} \in N_{k} / \Gamma_{k}$ for all $i \in N_{k}$. FDSU implies that

$\phi_{i}\left(v, \Gamma_{\mathcal{P}}\right)-\phi_{k}^{\mathcal{P}}\left(v_{\{i\}}^{k}, \Gamma_{\mathcal{P}_{\{i\}}^{k}}\right)=\frac{\phi_{i}^{\mathcal{P}}\left(v, \Gamma_{\mathcal{P}}\right)-\sum_{j \in N_{k}} \phi_{k}^{\mathcal{P}}\left(v_{\{j\}}^{k}, \Gamma_{\mathcal{P}_{\{j\}}^{k}}\right)}{n_{k}}, \quad$ for all $i \in N_{k}$

We have already determined $\phi_{k}^{\mathcal{P}}\left(v, \Gamma_{\mathcal{P}}\right)$ and $\phi_{k}^{\mathcal{P}}\left(v_{\{j\}}^{k}, \Gamma_{\mathcal{P}_{\{j\}}^{k}}^{k}\right)$, for all $j \in N_{k}$. So, Eq. (26) determines $\phi_{i}\left(v, \Gamma_{\mathcal{P}}\right)$ for all $i \in N_{k}$. 
INDUCTION HYPOTHESIS: Assume that the values $\phi_{i}\left(v, \Gamma_{\mathcal{P}}^{\prime}\right)$ have been determined for every $\Gamma^{\prime}$ with $\left|\Gamma^{\prime}\right|<\left|\Gamma_{k}\right|$.

INDUCTION STEP: Take a component $C \in N_{k} / \Gamma_{k}$. If $c=1$, then $C$ is a singleton and the payoff $\phi_{i}\left(v, \Gamma_{\mathcal{P}}\right)$ of the only player $i \in C$ is uniquely determined by (25). If $c \geq 2$, then there exists a spanning tree $\left.\widetilde{\Gamma} \subseteq \Gamma_{k}\right|_{C}$ on $C$ with the number of links $|\widetilde{\Gamma}|=c-1$. By UDL it holds that

$$
\Psi^{D L^{k}}\left(\phi^{k}\left(v, \Gamma_{\mathcal{P}}\right), \Gamma_{k}\right)=0
$$

Similarly to the proof of Theorem 2, due to the induction hypothesis, the $c-1$ equations (27) associated to the links $\{i, j\} \in \widetilde{\Gamma}$ together with (24) when $C=N_{k}$, form a system of $c$ linearly independent equations in the $c$ unknown payoffs $\phi_{i}\left(v, \Gamma_{\mathcal{P}}\right), i \in C$. Thus, for every $C \in N_{k} / \Gamma_{k}$, all payoffs $\phi_{i}\left(v, \Gamma_{\mathcal{P}}\right), i \in C$, are uniquely determined.

\section{References}

Aumann RJ, Drèze J (1974) Cooperative games with coalitional structures. Int J Game Theory 3:217-237 Béal S, Rémila E, Solal P (2012) Compensations in the Shapley value and the compensation solutions for graph games. Int J Game Theory 41:157-178

Borm P, Owen G, Tijs S (1992) On the position value for communication situations. SIAM J Discrete Math 5:305-320

Herings PJJ, van der Laan G, Talman AJJ (2008) The average tree solution for cycle-free graph games. Games Econ Behav 62:77-92

Kamijo Y (2009) A two-step Shapley value for cooperative games with coalition structures. Int Game Theory Rev 11:207-214

Khmelnitskaya AB (2010) Values for rooted-tree and sink-tree digraphs games and sharing a river. Theory Decis 69:657-669

Khmelnitskaya A (2014) Values for games with two-level communication structures. Discrete Applied Math 166:34-50

Kongo T (2011) Value of games with two-layered hypergraphs. Math Soc Sci 62:114-119

Meessen R (1988) Communication games. Master's thesis, Department of Mathematics, University of Nijmegen, The Netherlands (in Dutch)

Myerson RB (1977) Graphs and cooperation in games. Math Oper Res 2:225-229

Owen G (1977) Values of games with a priori unions. In: Henn R, Moeschlin O (eds) Essays in mathematical economics and game theory. Springer, Berlin, pp 76-88

Shapley LS (1953) A value for $n$-person games. In: Tucker AW, Kuhn HW (eds) Contributions to the theory of games II. Princeton University Press, Princeton, pp 307-317

Slikker M (2005) A characterization of the position value. Int J Game Theory 33:505-514

van den Brink R, van der Laan G, Vasil'ev V (2007) Component efficient solutions in linear graph games with applications. Econ Theory 33:349-364

van den Brink R, Khmelnitskaya A, van der Laan G (2016) An Owen-type value for games with two-level communication structure. Ann Oper Res 243:179-198

Vázquez-Brage M, García-Jurado I, Carreras F (1996) The Owen value applied to games with graphrestricted communication. Games Econ Behav 12:42-53

Zhang G, Shan E, Kang L, Dong Ya (2017) Two efficient values of cooperative games with graph structure based on $\tau$-values. J Comb Optim 42:462-482 